\title{
Dynamic GAN for High-Quality Sign Language Video Generation from Skeletal poses using Generative Adversarial Networks
}

\section{B Natarajan}

SASTRA Deemed University: Shanmugha Arts Science Technology and Research Academy

Elakkiya R ( $\nabla$ elakkiyaceg@gmail.com )

SASTRA Deemed University: Shanmugha Arts Science Technology and Research Academy https://orcid.org/0000-0002-2257-0640

\section{Research Article}

Keywords: Dynamic Generative Adversarial Networks (Dynamic GAN), Indian Sign Language (ISL-CSLTR)

Posted Date: August 9th, 2021

DOI: https://doi.org/10.21203/rs.3.rs-766083/v1

License: (c) This work is licensed under a Creative Commons Attribution 4.0 International License.

Read Full License 


\title{
Dynamic GAN for High-Quality Sign Language Video Generation from Skeletal poses using Generative Adversarial Networks
}

\author{
Natarajan $B^{a}$, Elakkiya $R^{b}$ \\ ${ }^{a}$ Research Scholar, ${ }^{b}$ Assistant Professor \\ School of Computing, SASTRA Deemed To Be University, Thanjavur, Tamilnadu, India \\ \{elakkiyaceg@gmail.com\}
}

\begin{abstract}
The emergence of unsupervised generative models has resulted in greater performance in image and video generation tasks. However, existing generative models pose huge challenges in high-quality video generation process due to blurry and inconsistent results. In this paper, we introduce a novel generative framework named Dynamic Generative Adversarial Networks (Dynamic GAN) model for regulating the adversarial training and generating photorealistic high-quality sign language videos from skeletal poses. The proposed model comprises three stages of development such as generator network, classification and image quality enhancement and discriminator network. In the generator fold, the model generates samples similar to real images using random noise vectors, the classification of generated samples are carried out using the VGG-19 model and novel techniques are employed for improving the quality of generated samples in the second fold of the model and finally the discriminator networks fold identifies the real or fake samples. Unlike, existing approaches the proposed novel framework produces photo-realistic video quality results without using any animation or avatar approaches. To evaluate the model performance qualitatively and quantitatively, the proposed model has been evaluated using three benchmark datasets that yield plausible results. The datasets are RWTH-PHOENIX-Weather 2014T dataset, and our self-created dataset for Indian Sign Language (ISL-CSLTR), and the UCF-101 Action Recognition dataset. The output samples and performance metrics show the outstanding performance of our model.
\end{abstract}

\section{Introduction}

Sign language greatly improves the communication skills of the deaf-mute community as well as explores the needs and emotions of such people. Sign languages are highly structured, visual conveying, and multi-channel based one, expressed via gestures and utilizes human body parts such as hands, face, eyes and gaze movements [42]. These components are usually termed as manual components for hands actions and non-manual components for facial and mouth expressions [8]. In most countries, sign languages are developed based on culture, traditions, and surroundings. These variations are referred to as multimodal sign gestures for multilingual sentences. Recognition and translation of such different variations in sign gestures create numerous challenges to the researchers and require expert skills in computer vision and artificial intelligence domains. Research studies on sign language recognition and translation attained wider attention around the globe. The development of such systems assists normal people to easily communicate with deaf-mute people to provide training and education services. Automation of these translation processes with the help of high-power computing devices will rise the digital technology advancements to the next level. The growth of hardware technology handles such high-level computing tasks using GPU devices. The proposed work emphasizes the translation of skeletal pose into sign language videos using deep generative networks. The proposed model creates a single unified framework to process multimodal skeletal poses and translate them into human-based sign gesture images and combines the gesture image sequences for video generation. The earlier generative models using deep learning approaches have reached various milestones by producing impressive results in image and video generation. The existing approaches like auto-encoder and its variants VAE and CVAE $[23,24]$ generate images with blurred effects. The quality of generated results does not 
comply with the expectations. The recent advancements in generative adversarial networks have been attained wider attention among researchers for developing various applications like synthesizing medical images [25,42], text-to-image translation [17], video analytics [26], and creating human images that do not exist in the world [12]. This powerfulness of the GAN models directs the researchers to develop efficient models to generate high-quality images or videos.

However, the processing of a large number of images or videos and producing new images or video potentially requires high expert skills. Research and development of such models explore the capability of generative networks to the next level. Predicting the future frames [33], video completion [33] and video generation [36] showcases the current improvements in GAN model development. These advancements in the GAN techniques can be applied to generate high-quality photo-realistic sign videos from skeletal poses for the betterment of the deaf-mute community. In this paper, the development of the dynamic GAN model is divided into various folds. In the first fold, the mapping of skeletal poses and ground truth images took place, then the generator network generates human-based sign gesture images. In the Next fold, we apply the Image Classification using VGG-19 and Image alignment Techniques. Further, we apply deblurring techniques for improving the image quality. The generation of intermediate frames for connecting the sequences of gestures has been carried out in the proceeding steps. Finally, the discriminator network produces a photorealistic high-quality sign Video generation process by checking the reality of images. In the case of fake images, the model redirects the results to the generator to undergone fine-tuned training for generating high-quality results.

The introduction of the Generative Adversarial Network (GAN) framework [1] greatly scaled up the growth of the deep generative model to generate high-quality images or videos. These models have attained greater attention among researchers to develop powerful models for high-quality image or video generation. The two networks of the GAN model play the minimax adversarial game competently to produce high-quality videos. The generator networks aim to produce images similar to the real ones from random noise vectors. The discriminator network classifies the real and fake images intelligently. Based on such classification, the generator network fine-tunes its training performance to produce good quality videos which mimic real videos. In a basic model of GAN [1], the Multi-layer perceptron-based fully connected layers and the activation function ReLU is applied in the generator network side and max out activations are applied in the discriminator network. The model has been evaluated using the benchmark datasets such as the MNIST handwritten digits dataset and Multi-class images based CIFAR-10 dataset. The basic model has been upgraded to various levels to achieve greater emoluments in multiple domain datasets. The extended model known as DCGAN [51] was implemented for stabilizing the training process in the generator end using deep CNN approaches. The extended version of the basic GAN model is called as conditional GAN [2] model which applies conditioning on class labels to produce high sharpened results in the generation of new digits images using the MNIST handwritten digits dataset depicted in Fig. 2.

The InfoGAN models [60] utilizes the latent space for encompassing semantic label information with real images for generating improved quality images. Auxiliary Classifier GAN [45] model employs the conditional GAN for conditioning the class labels and adds the auxiliary models for reconstructing the class labels. The development of StackGAN models [18] uses hierarchical stacked approaches combined with conditional GAN networks for generating images from text. This model follows two stages of development. In the first level, it produces images based on the text by applying conditioning on text data, which results in the low-resolution images. In the second stage, this model improves the results by conditioning 
low-resolution images and text. Finally, it produces high-resolution images. Similar to the features of GAN models the context Encoders [40] uses adversarial approaches to generate conditioned images by applying conditions on its surrounding parts. This model uses reconstruction loss and adversarial loss approaches to yield sharpened results. The pix2pix generative model [5] extends the basic framework of GAN models to uplift its performance in the image to image translation tasks. This model incorporates the U-Net framework in the generator phase and applies the PatchGAN framework in discriminator phases for supporting different domain applications like generating photos from semantic labels, black and white images to color image translation, edges to real image conversion, day and night scene translation, photo editing and creating new effects.

The recent advancements in generative adversarial networks have been greatly improved the GAN performance to the next level by generating photo realistic images. The variant of the GAN network referred to as Wasserstein generative adversarial network (WGAN) [46] introduces critic which alters the training steps for updating the discriminator network. The Wasserstein loss functions are introduced in this model for improving the output image quality. The cycle-consistent generative adversarial network (CycleGAN) [22] has been developed for performing the image-to-image translation tasks without using conditioned target images. This model follows reversible approaches to produce one form to another by utilizing the cycle consistent approaches. The Progressive GAN [47] models emerged with new approaches for training the generative networks. This model adds extra layers to stabilize and progressively improve the performance of the model and yields unprecedented quality images. The BigGAN models [48] improves the image quality by scaling up the existing conditioning models and changes the training parameters. The use of the truncation trick in latent space highly boosts the model performance. The StyleGAN [27] models use different latent space embedding techniques to synthesis the images. It controls the features of output images by inputting the latent code with different points.

Although a lot of advancements were proposed in various papers, there is a great demand for the development of a single unified generative framework to produce high-quality images or videos for multiple domains. The proposed dynamic GAN models introduce novel techniques to effectively train the generator models and applying various image processing techniques to improve the generated results quality in terms of variation, texture, edge sharpening, and diversity which lead to the production of photo-realistic sign videos from skeletal poses. The intermediate frame generation and video completion approaches lead the discriminator network to classify the generated videos as the real ones. From a development point of view, the translation of human skeletal pose images into sign videos incurs huge challenges in model development and also needs to address the bottlenecks of conventional sign language recognition tasks. The execution order of sign gestures is highly differing from the word order of spoken language sentences. To address this issue, we introduce novel image alignment techniques for arranging the sign gesture images. The selection of relevant sign images and generating the in-between frames requires much attention for the video completion process. In the output videos, we consider the video quality by avoiding the collision of sign gestures. In addition to this, preserving the naturalness and identification of epenthesis movements, resolving the gesture ambiguities, co-articulation issues, and ill-posedness [16,43] are also considered for generating good quality results. The continuous recognition of the dynamic changes of sign gestures related to spoken sentences poses huge challenges. On the other hand, processing large-scale datasets with multimodal features. We need to consider all these challenges for developing the powerful framework. 
We have aimed to achieve the following objectives as an outcome of this work.

- We develop a novel GAN framework for generating photo-realistic high-quality sign language videos by processing the skeletal pose images and ground truth images.

- We evaluate the model performance using different benchmark datasets such as RWTH-PHOENIX-Weather 2014T dataset, the ISL-CSLTR dataset, and the UCF-101 Action recognition dataset.

- We build a single unified architecture for generating videos in multiple domain cases such as action recognition, analysis of human behavior in public, and monitoring the activities of people in a crowded environment.

Further discussions about this work are planned as follows. The existing developments present in generative models were discussed in section 2, the proposed system and implementation details were discussed in section 3. In section 4, the experimental results on benchmark datasets are discussed and finally the conclusion and future work part summarize the entire work.

\section{Related Works}

Research studies on high-quality video generation using latent space data points have been identified as a challenging task since the last centuries. Due to the mode collapse, robustness, instability, scalability, and inconsistent results, the earlier approaches on video generation produces low-resolution videos. The generation of high-quality photo-realistic videos requires a lot of supervision on the unsupervised distribution of data points present in the latent space. Each data points placed in a latent space contribute some portion of the video to establish the sequential relationship that exists between sign gestures, helps to automate the high-quality video generation process. The earlier approaches [1-10] discusses the generation of images or videos from noise vector by randomly selecting some data points. Due to a lack of efficient training process and various factors, these models mostly produce blurry and inconsistent results. Although, the latent space provides necessary information about existing data points, still needs some efficient mechanism to enhance the selection of data points to produce high-quality photorealistic videos. The emergence of GAN models handles such image or video generation tasks efficiently using generator and discriminator networks. The production of sign videos needs much attention in selecting latent space data points due to the variants of input sentences and dynamic changes in selecting sign gesture images and incorporation of spatial and temporal features to produce videos. In order the preserve the consistency in output quality, we need to investigate various techniques for producing finegrained human perceptual results.

In general, machine learning models can be classified as discriminative and generative models. Usually, the discriminative models work well for classification-based tasks like spam detection in email. On the other hand, generative models are powerful in creating samples based on underlying data distribution. The variants of generative models are parametric and non-parametric approaches. Parametric approaches are highly used for image reconstruction purposes, whilst non-parametric approaches are highly used in text synthesis [10] and speech synthesis [9] processes. Learning the low-dimensional details of data distribution supports the image reconstruction process [28]. Mostly the popular models such as deep auto-encoders [23, 24] or the generative model known as Restricted Boltzmann machines (RBM) [18-22] were primarily used for generating images. Due to higher complexity in generating images, these techniques are found as less effective one. The advent of variational auto-encoders (VAE) models [23] resolves this issue by adopting a variational sampling based approaches, but which are limited to small scale datasets such as MNIST. The generation of human recognizable images with rich content the VAE model were stepped in new advancements [23,24]. 
GAN models are introduced by the author [1] discuss the adversarial training process by placing two players (generator and discriminator) in a game of competing with each other using minimax approaches. The maximization of the first player score will minimize the second player score vice versa. This discriminative process aims to produce handwritten images, faces, and objects. The primary model was targeted to achieve global optimum by matching the produced results with original data. This model produces blurred results that need to be improved using conditional-based approaches and inference mechanisms. This model considers only a specific portion of the data distribution, divergence, and oscillation nature tends to training difficulties. The conditional-based GAN models [2] apply conditional probabilistic rules on both the generator and discriminator sides to generate improved results. These models apply conditions on some portion of data. These models apply conditions on class labels over handwritten digit datasets and highly capable to learn the multimodal models. The condition-based predictive distribution produces good results over the learned distribution of data and results in the deterministic relationship between input and output. The conditional GAN models lead the development of image to image translation models [3], face generation [4], face aging [5], domain adaption models for alignment of multimodal features $[63,66]$, image captioning [24], machine translation [13], Text to Image synthesis [9,10].

The least-square GAN model [15] was developed to address the vanishing gradient issues persist with discriminator classifier by employing the decision boundary-based penalization strategies. Although, this model produces comparable results over the regular GAN model, requires much improvement for generating real images by automating the penalization steps. The 3CATN [64] model is proposed to address the challenges in adversarial training that still need to be improved for unsupervised learning tasks. The StackGAN[18] models follow two-stage approaches for translating the text scripts into real images. In the first stage, it creates outline-based low-resolution images, after applying condition-based augmentation techniques it produces photorealistic results. However, these models fail to perform well on real-time image generation tasks due to model collapse and instability issues. The author [19] was developed Laplacian Pyramid-based GAN model (LPGAN). This model uses down sampling and up sampling methods to construct high-quality images places high complexity in the simple image generation process and bottlenecks with sequence images in video generation tasks. The Large Scale GAN models [48] are developed to handle huge classes of imagenet based natural images using orthogonal regularization techniques. Although this model suits large-scale training, model collapse may occur and results in low-resolution images or videos. The author[67] proposed DiscoGAN models for identifying and classifying different domain objects and applied style transfer mechanisms to change the objects of one domain to another domain without losing identity key points. In [50] author introduced the chair image generation using CNN Techniques. The Laplacian pyramid GAN models [19] are developed to produce fine-grained images from coarse-grained inputs but fail to generate stable results. However, the DCGAN model provides promising results, requires the conditioning of class labels at each time step. The author [49] implemented the new approach for predicting the future frames by applying conditions on the previous frames. The author [58] introduced the MoCoGAN model for dealing with the motions and content features in the video generation process. The random vector sequences are trained properly for generating sequence of frames in order, produces high quality videos.

The FUNIT model [71] generates multiple domain images few-shot based image translation approaches. This model requires fully class labelled image datasets and fails to handle the dynamic generation of frames for image or video creation. In parallel work, the author [27] combines multimodal and multi-domain translation by employing the manipulation schemes in latent spaces. These models limit their performance for learning various styles of multi-mapping translation tasks. In [20] author developed the StarGAN model using one generator and one discriminator networks for the translation of image to image tasks and supports scalable services. This model aims to provide high-quality images owing to the generalization and multitasking capabilities. Besides, the simple mask vector concepts enrich 
the model performance to operate well in multiple domains. Since this model handles multiple domain inputs, fails to incorporate the distinguishable features. The revised version StarGAN v2 [21], produces improved results in multiple domains and handles diversity and scalability issues very well. Since these models are implemented for working with inter-domain and intradomain based applications, need to be enhanced for video generation in multiple domains by adopting different features. The GAN model and the advancements have been found significantly important for learning the structure of deep generative models to generate images or videos similar to real-time data. However, the persist of instability and mode collapse issues with the generated results, the Evolutionary GAN model [41] addresses these issues by employing different adversarial training methods and mutation operations in both generator network and discriminator network.

In [51], the author investigated the CNN-based generative network for learning unsupervised feature representations, but this model needs to improve the learning of latent spaces to avoid mode collapse issues in generated results. We used an open pose library [52] in our model for extracting skeletal poses from real images. The skeletal poses are highly useful to train the generator network to generate images similar to real ones. The existing models for estimating the human poses consider various parameters present in the input images. To find various gesture actions performed by human body components local features identification and extraction techniques are used. Many of the researchers are proposed different techniques such as graphical models [53], non-tree models [54]. In many cases, CNN [55] found a highly very useful approach in pose estimation. Detecting multiple people poses in a single image creates higher complexity to the existing model. The open pose model [35] gives a solution to this problem using part affinity fields. Table 1 describes the detailed information of various existing generative frameworks.

\begin{tabular}{|c|c|c|c|c|}
\hline Author & Model & Dataset & Metric & Image/Video \\
\hline $\begin{array}{l}\text { Goodfellow et al.(2014) } \\
\text { [1] }\end{array}$ & GAN & MNIST, TFD,CIFAR-10 & log-likelihood estimate & Image \\
\hline $\begin{array}{l}\text { Salimans et al. (2016) } \\
\text { [3] }\end{array}$ & Improved GAN & MNIST, CIFAR-10, SVHN & Test error rate & Image \\
\hline Ma et al. (2017) [6] & $\begin{array}{l}\mathrm{PG}^{2} \text { (Pose Guided } \\
\text { Person Generation } \\
\text { Network) } \\
\end{array}$ & Deep Fashion, Market-1501 & SSIM, IS & Image, Video \\
\hline Siarohit et al. (2018) [7] & Deformable GAN & Deep Fashion, Market-1501 & SSIM, IS & Image, Video \\
\hline Mirza et al. (2014) [2] & Conditional GAN & $\begin{array}{c}\text { MNIST, MIRFlickr 25,000 } \\
\text { dataset }\end{array}$ & log-likelihood estimate & Image \\
\hline Isola et al. (2017) [5] & Pixel GAN & Cityscape dataset & FCN Score & Image \\
\hline Shishir et al.(2020) [11] & EsharaGAN & IsharaLipi Dataset & IS & Image, Video \\
\hline Stoll et al. (2020) [31] & $\begin{array}{l}\text { Pix2pixHD + VAE- } \\
\text { GAN }\end{array}$ & SMILE sign language dataset & SSIM, PSNR, MSE & Image, Video \\
\hline Zhao et al. (2018)[61] & VariGANs & MVC, Deep Fashion & SSIM, IS & Image, Video \\
\hline $\begin{array}{l}\text { Tulyakov et al.(2018) } \\
\text { [58] }\end{array}$ & MoCoGAN & $\begin{array}{c}\text { Taichi video clips, MUG Facial } \\
\text { Expression dataset }\end{array}$ & $\begin{array}{l}\text { Motion Control Score (MCS), } \\
\text { IS, Average Content Distance } \\
\text { (ACD), user preference score }\end{array}$ & Image, Video \\
\hline $\begin{array}{l}\text { Vondrick et al.(2016) } \\
\text { [57] }\end{array}$ & VGAN & Flickr & user preference score & Image, Video \\
\hline Saito et al.(2017) [9] & TGAN & $\begin{array}{l}\text { Moving MNIST, UCF-101, Golf } \\
\text { scene dataset }\end{array}$ & $\begin{array}{l}\text { IS,GAM (Generative } \\
\text { Adversarial Metric ) }\end{array}$ & Image, Video \\
\hline $\begin{array}{l}\text { Arjovsky et al. (2017) } \\
\text { [46] }\end{array}$ & WGAN & LSUN Bedrooms dataset & Earth Mover (EM) distance & Image, Video \\
\hline $\begin{array}{l}\text { Radford et al. (2015) } \\
\text { [51] }\end{array}$ & $\begin{array}{c}\text { Deep Convolutional } \\
\text { GAN } \\
\end{array}$ & CIFAR-10, STL, LSUN & Classification Accuracy & Image, Video \\
\hline Wang et al. (2016) [62] & $\mathrm{S}^{2} \mathrm{GAN}$ & $\mathrm{NYUV}^{2}$ dataset & Classification Accuracy & Image, Video \\
\hline He et al. (2018) [32] & VideoVAE & $\begin{array}{c}\text { Chair CAD, Weizmann Human } \\
\text { Action dataset, YFCC, MIT } \\
\text { Flickr } \\
\end{array}$ & IS & Image, Video \\
\hline Wang et al. (2019) [35] & P2P video generation & $\begin{array}{l}\text { Moving MNIST dataset, Human } \\
\text { 3.6M, BAIR Robot pushing } \\
\text { dataset, Weizmann Action } \\
\text { dataset, }\end{array}$ & PSNR, SSIM,MSE & Image, Video \\
\hline Aigner et al. (2018) [59] & FutureGAN & $\begin{array}{c}\text { Moving MNIST, Cityscape, } \\
\text { KTH Action } \\
\end{array}$ & PSNR, SSIM,MSE & Image, Video \\
\hline Karras et al. (2017) [47] & PGGAN & CIFAR-10,CelebA,LSUN & IS & Image, Video \\
\hline
\end{tabular}

* PSNR - Peak Signal-to-Noise Ratio, SSIM - Structural Similarity Index Measure, IS - Inception Score, MSE-Mean Squared Error, FCN - Fully-Convolutional Semantic Segmentation Network

Table 1. Comparison of various generative models 


\section{The proposed system}

\subsection{GAN}

The first GAN model was introduced by the author [1] in the year 2014. The basic architecture of this model comprises the generator $(\mathrm{G})$ and discriminator (D) networks. The generator network creates fake images similar to input images by tracking the data distribution. The discriminator classifies the fake and real images using probability functions. It was modeled like playing two players-based minimax games. Each network aims to increase its score by decreasing other network scores. Finally, it results in the production of high-quality images.

$$
\min _{\text {Gen }} \max _{\text {Dis }} V(\text { Dis, Gen })=\mathbb{E}_{x} \sim p_{\text {data }}[\log \operatorname{Dis}(x)]+\mathbb{E}_{z \sim p_{z}(z)}[\log (1-\operatorname{Dis}(\operatorname{Gen}(z)))]
$$

In equation (1), $p_{\text {data }}$ represents the real images and $p_{z}$ denotes the noise vector values. We use the basic GAN network models in our work for generating videos. The generator and discriminator networks are fine-tuned to produce photo-realistic high-quality videos.

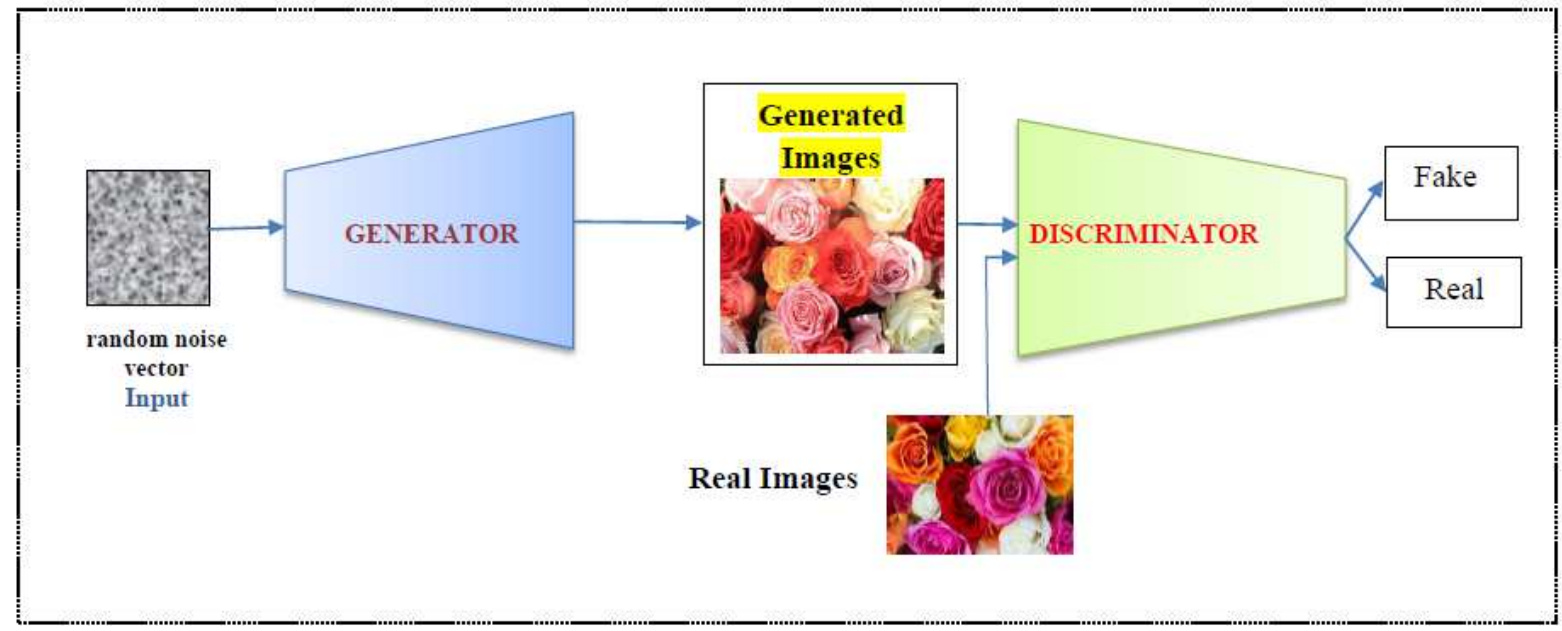

Figure 1. Basic GAN Model

\subsection{Conditional GAN}

The conditional GAN models [2] have been achieved tremendous success in image or video generation. These models are evidenced as powerful ones in many GAN variant models $[5,14,45]$ to produce high-quality images. It applies conditioning on the class labels, assists the generator network to produce sharpened results by considering angles and orientation. Equation (2) describes the cGAN model.

$$
\min _{G e n} \max _{\text {Dis }} V(D, G)=\mathbb{E}_{x, y} \sim p_{\text {data }}[\log \operatorname{Dis}(x \mid y)]+\mathbb{E}_{z \sim p_{z}(z)}[\log (1-\operatorname{Dis}(\operatorname{Gen}(z \mid y)))]
$$

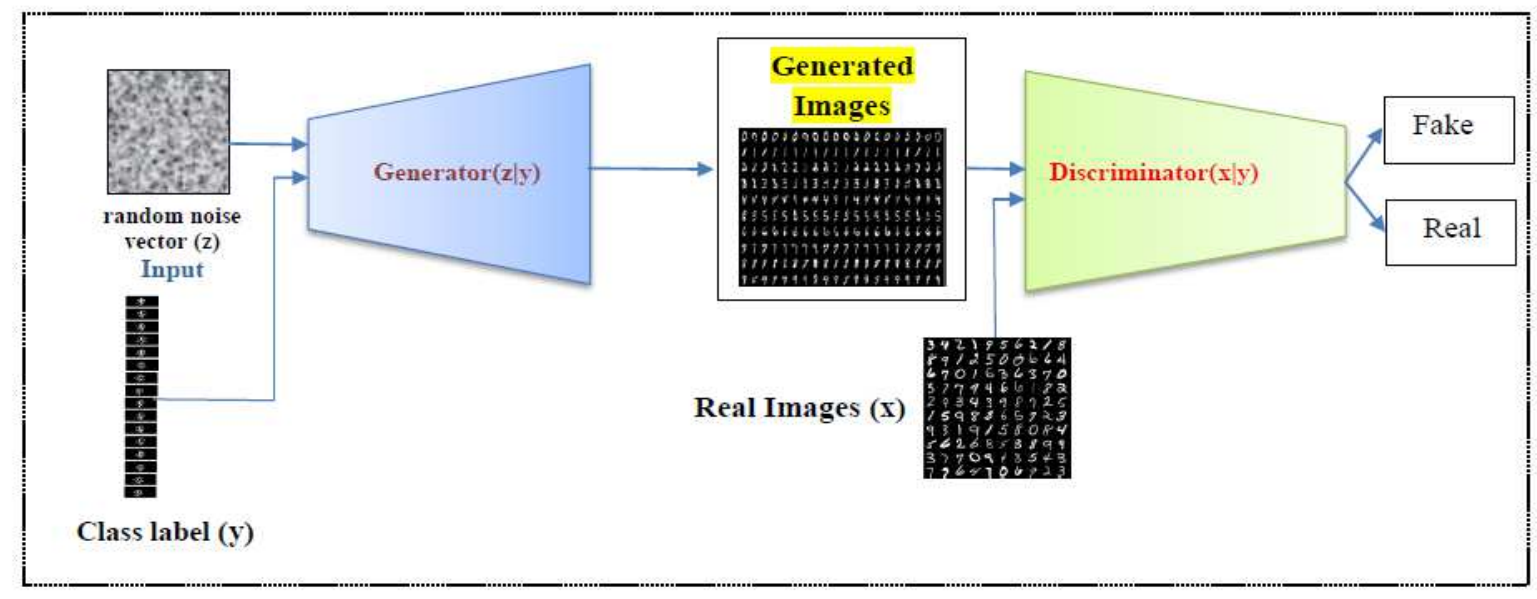


We have incorporated the conditional GAN method with our proposed model for applying condition on sign glosses (class labels) to generate sharpened images with clear sign gesticulations in generated images.

\subsection{OpenPose}

The OpenCV-based OpenPose library [52] is mainly developed for human pose estimation in different environments like playground, meeting, dancing, street walking and interactions with others. The OpenPose techniques are developed by Carnegie Mellon University (CMU) researchers for aiding the implementation of applications to track human actions, movements, behavior, and interactions in real-life environments. It detects the human body parts like the head, hands, limbs, and foot points. It helps to identify the activity and pose orientation of a human in images or videos by plotting color lines over the human images. This can be extensively used to track human activity in public or highly secured environments. This model learns the two-dimensional poses estimation from human body parts by adopting Part Affinity Fields approaches described in equation (3). This technique follows bottom-up approaches that tend to produce improved results over earlier methods. It also detects key points in vehicle images and predicts the poses of hidden components in the human body. The overall pipeline of the open pose model comprises various folds. In the first fold, the input RGB color image gets processed for producing estimation of key points in 2D anatomical positions. For this estimation, the first ten layers of the popular CNN model known as VGG19 are used. In the second fold, using part affinity fields and confidence maps the relationship association of body parts was identified. With the help of greedy inference techniques, the final poses are extracted. We used double blending commands to extracted skeletal poses alone from the sign videos for further processing.

$$
\boldsymbol{L}_{v}^{*}=\frac{1}{n_{v}(\boldsymbol{P} \boldsymbol{t})} \sum_{u} \boldsymbol{L}_{v, u}^{*}(\boldsymbol{P} \boldsymbol{t})
$$

Where $n_{v}(\boldsymbol{P})$ denotes at point $\mathrm{Pt}$ the values of vector for $\mathrm{u}$ number of humans.

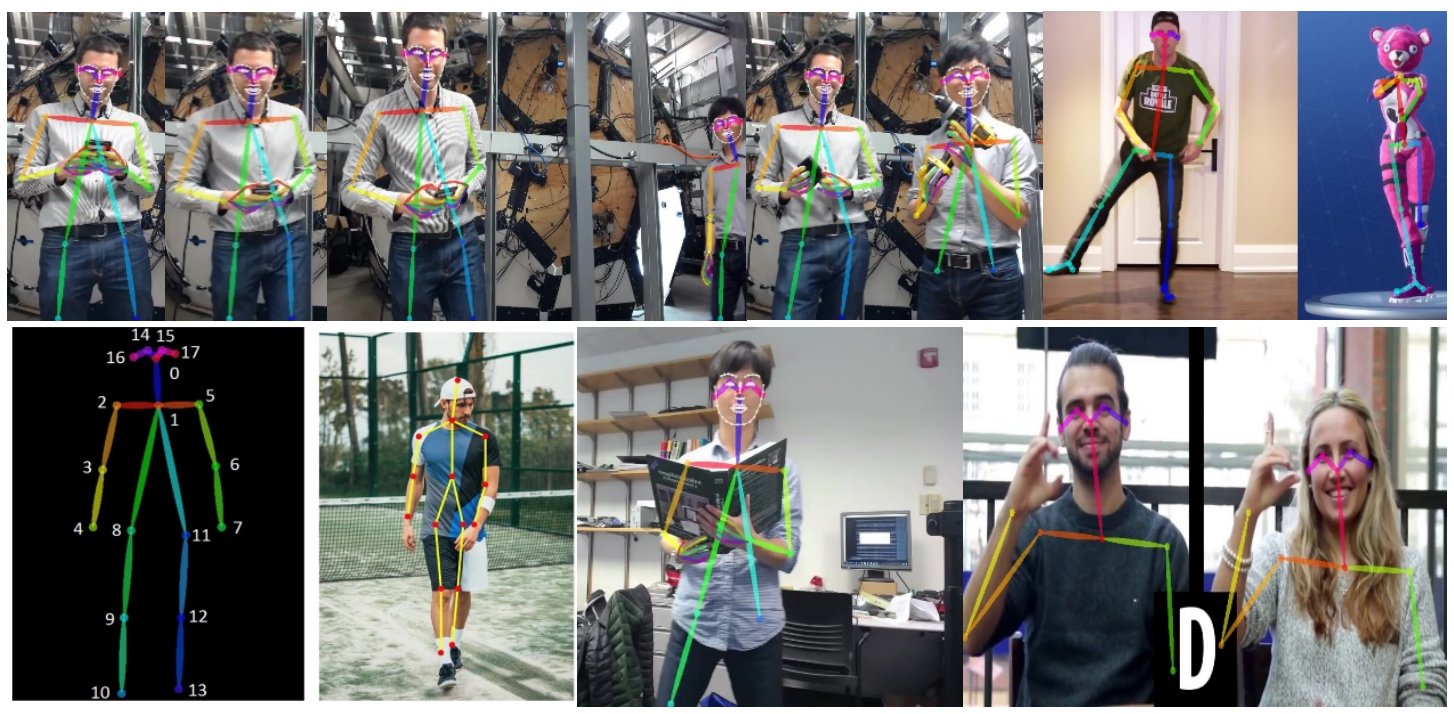

Figure 3. Sample pose estimation results of open pose library

We use the open pose library in our work for extracting skeletal poses and key points from sign gestures of multiple signers. These extracted poses and key point helps to detect the movements of signs from one form to another in continuous sign cases. From which, we can modify the key points to create multiple views of sign gestures using the methods proposed in [61]. The computation of affine transformation helps to identify the target pose feature maps. 
During the forward pass, the set of local affine transformations takes place to achieve the desired global pose-based deformation results. The coarse image generation using variants of Bayesian approaches provides a good approximation of conditional log-likelihood estimation and produces low-resolution images. The fine image generation approach uses generative networks to improve the quality of images. We use affine transformation, coarse image generation, and fine image generation techniques to produce high-quality images by referring to the skeletal poses and ground truth.

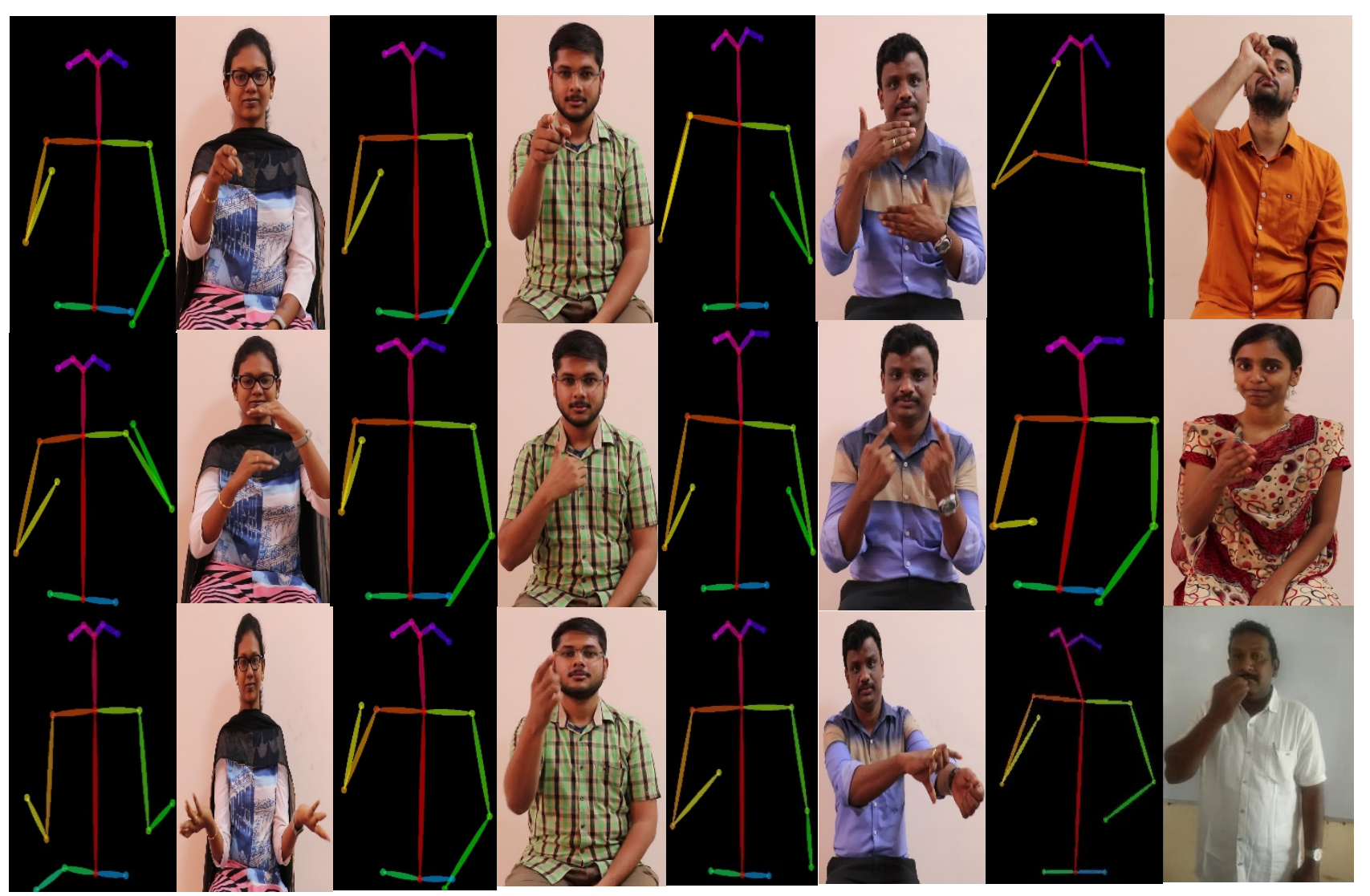

Figure 4. Sample pose estimation results of open pose library for ISL-CSLTR dataset

The improvements in open pose versions highly focus the possible failure cases like detecting poses in overlapping parts, presence of statues, various objects, and animals, misclassification of humans in highly crowded environments. These models train the machine to understand the interactions of humans in environments and estimates accurate poses. The model was evaluated using three datasets (i) MPII human multi-person dataset, (2) COCO keypoint challenge dataset (3) foot dataset, MPII human multi-person dataset consists of a total of 5602 images categorized as 3844 training images and 1758 testing images. The open pose library efficiently detects 14 body parts in such multi-human-based images or videos. The COCO dataset results showcase the improvements of the open pose models by estimating 17 features. The foot dataset has experimented with open pose models for handling the failure cases which perhaps due to the variations in human images, occlusions, and hidden cues.

\subsection{The proposed system}

The generator network used in our proposed model uses random noise vector values which are conditioned on sign glosses based class labels to generate sign images. The generated results are classified according to class of sign glosses group using the VGG-16 framework. Further, we apply intermediate frame generation techniques to create intermediary frames between sign gestures. The creation of intermediary frames correlates the sequence of actions between signs to explore the real actions and changes. The noise present in the images are cleaned using 
deblurring approaches. The pixel normalization techniques and video completion techniques are used for smoothening the final results. We employ video completion techniques proposed in [33] for generating intermediate frames between the sign images to synthesis the sequences of sign gestures as real one.

The perceptual loss and contextual L1 losses are combined to predict the intermediary frames between two sign gestures. The equation (4) describes the computations.

$$
\hat{z}=\underset{z}{\operatorname{argmin}}\left\{\operatorname{Loss}_{\text {context }}(z \mid I)+\alpha \times \operatorname{Loss}_{\text {percept }}(z)\right\}
$$

This optimization strategy produces fine grained results for effective video generation. These results are fed into the discriminator network to analyze the realism of generated results. The discriminator network classifies the real and fake samples. In case of fake, it iterates the model training to improve the learning performance of the model. Figure 3 explores the detailed architecture of the proposed dynamic GAN network.

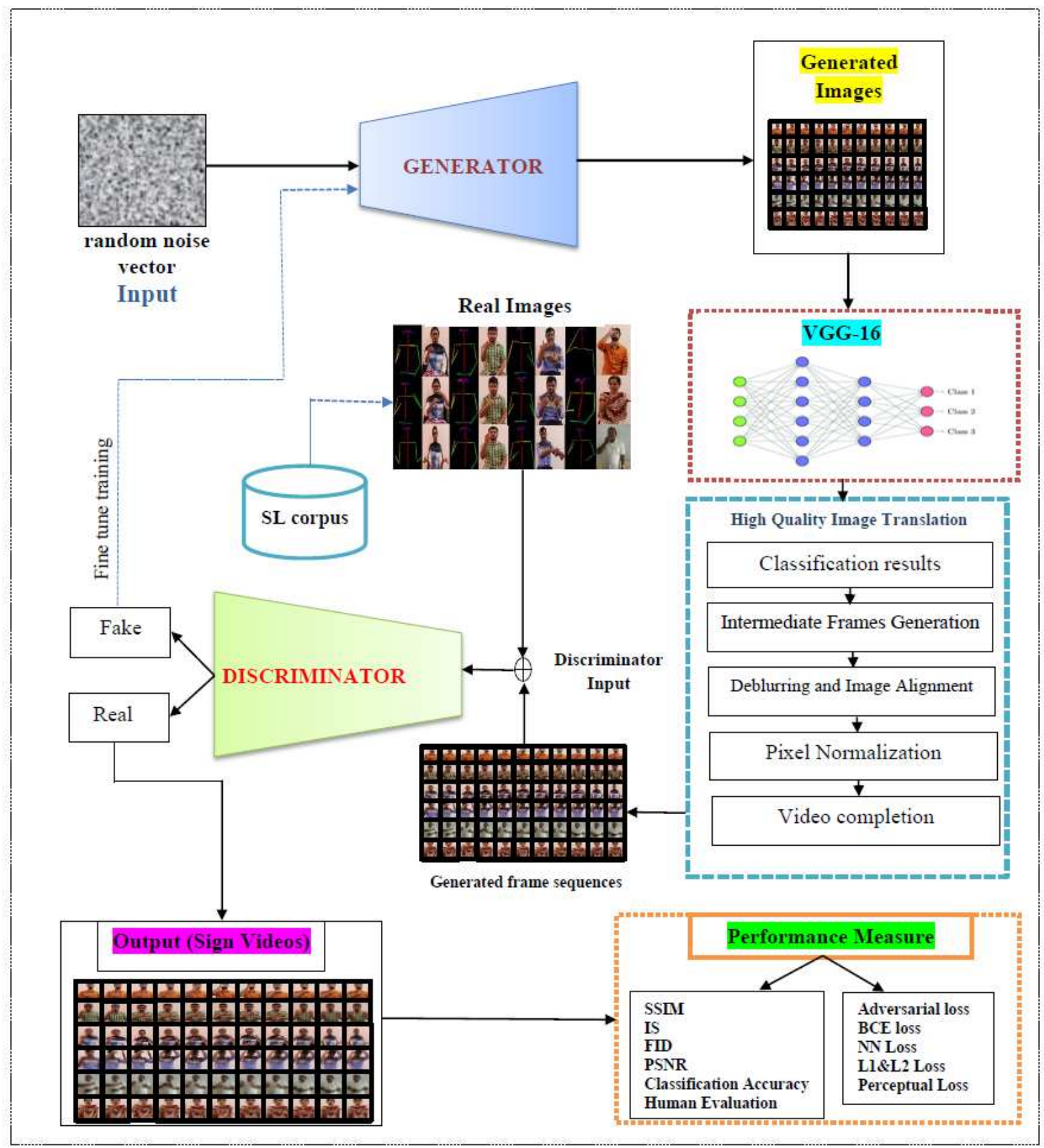

Figure. 5. The Proposed Dynamic GAN Network Architecture 
The proposed system functions are elaborated detail in algorithm (1) named as high quality video generation using dynamicGAN model, in which random noise vectors $\left(Z_{i}\right)$ are given as input to the generator network G. The training process will undergo several times based on input length and the final results fed into the discriminator network. The discriminator network (D) classifies the real and fake samples. Based on the training process, the quality of generator is improved to generate high quality images similar to real images.

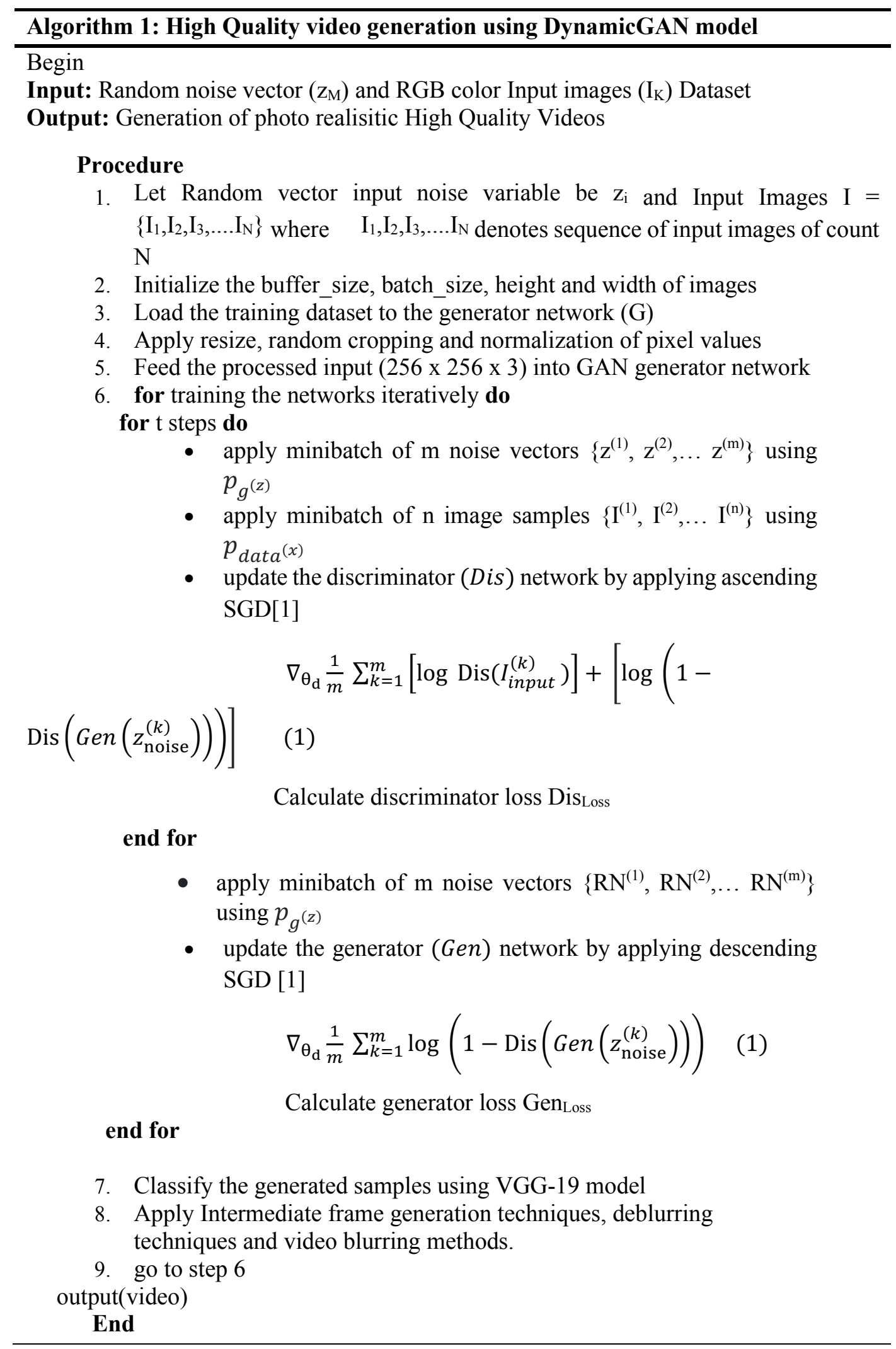




\subsection{The Generator Network}

The Generator Network generates sign images using conditional GAN (2) methods which are conditioned in class labels of sign glosses. The Generator loss is a sigmoid crossentropy loss of the generated images. We also include L1 loss which refers to the mean absolute error between the generated image and the target image. This allows the generated image to become structurally similar to the target image. Figure (7) explores the loss computation of the generator network. The layer wise details are plotted in figure (6).

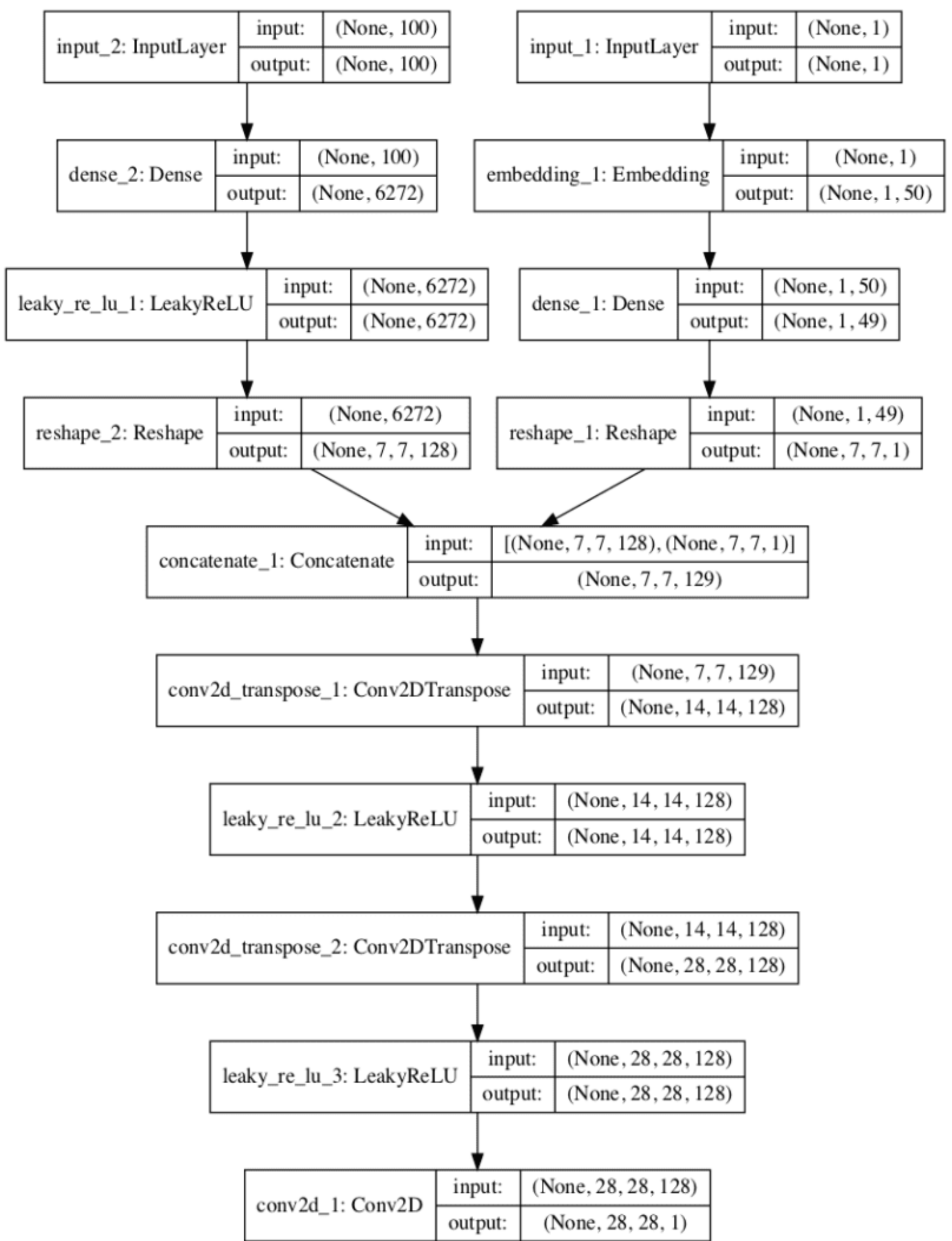

Figure 6. Layer details of Generator network 

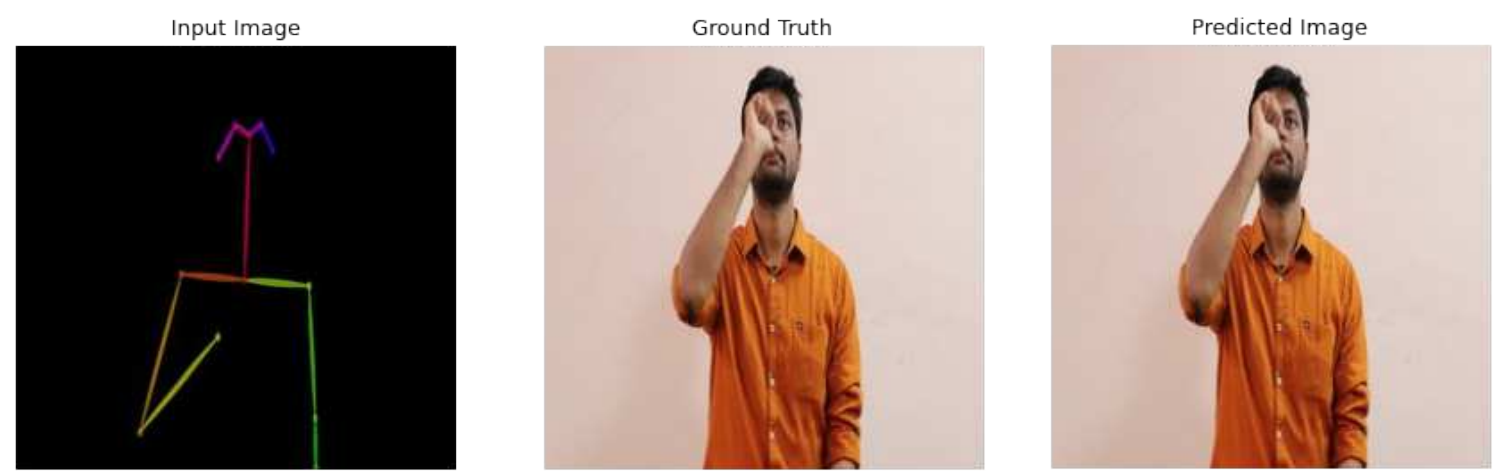

Figure 7. The sample image generation from skeletal poses using generator network

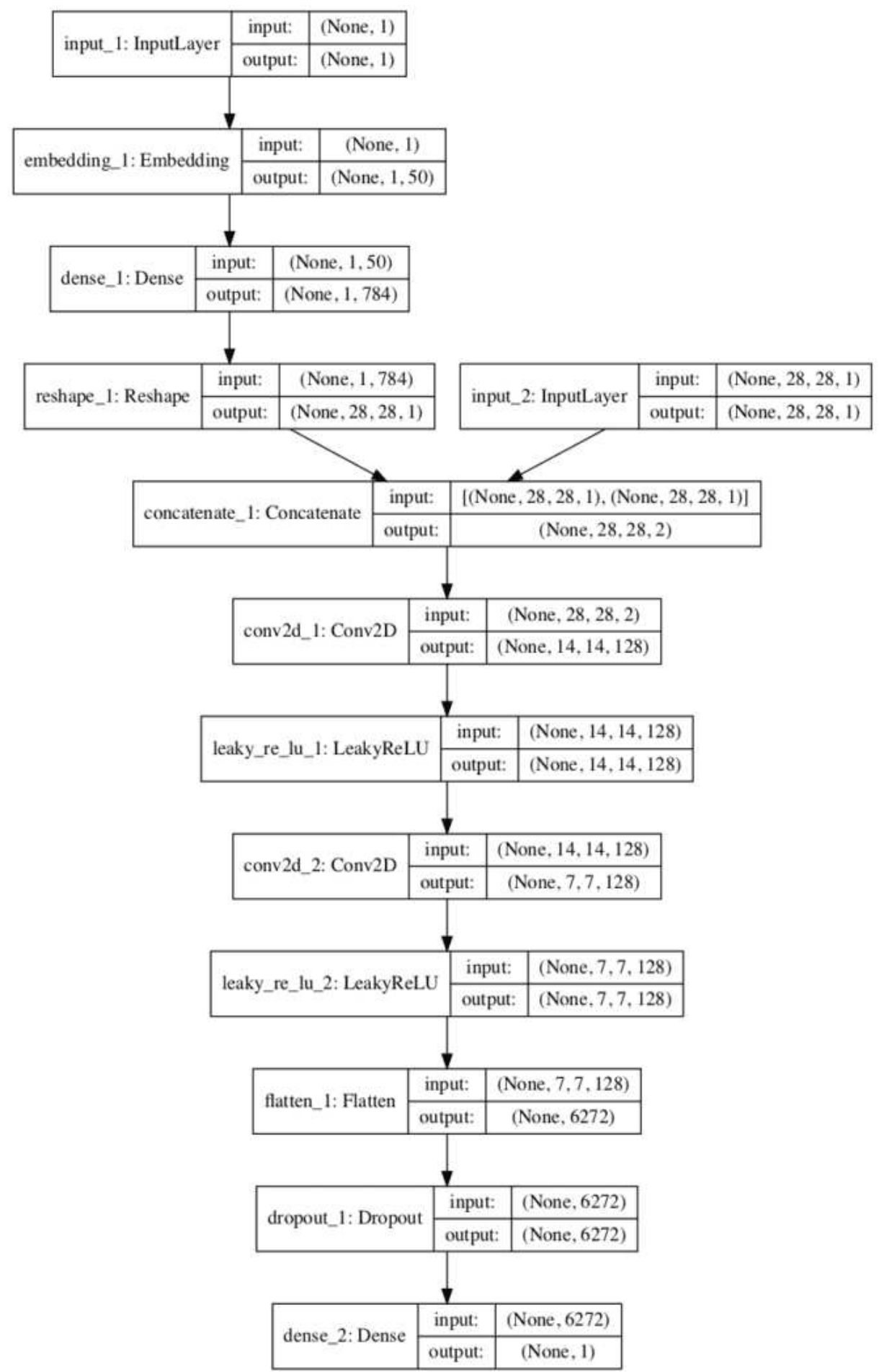

Figure 8. Layer details of Discriminator network 


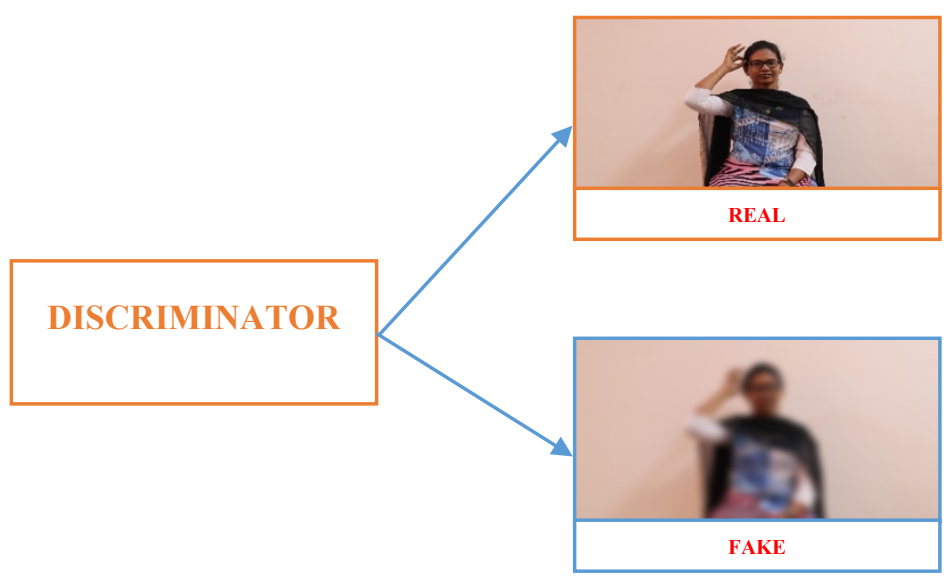

Figure 9. The discriminator classification of real and fake samples.

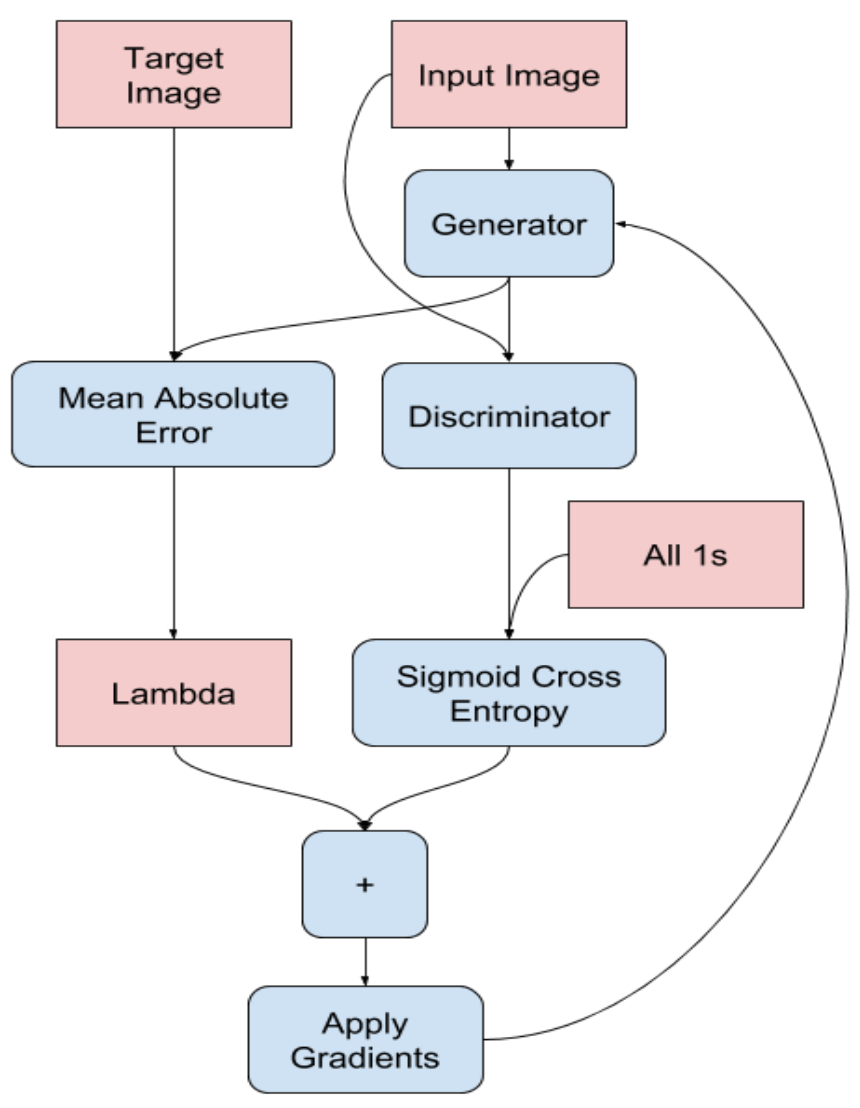

Figure. 10. The Training Procedure of Generator Network

\subsection{The Discriminator Network}

The Discriminator Network uses PatchGAN approaches. Each block in the discriminator is the Convolution layer, Batch normalization layer, and the activation function Leaky ReLU. The shape of the output after the last layer is (batch_size, 30, 30, 1). Each 30x30 patch of the output classifies a 70x70 portion of the input image. The discriminator receives 2 inputs. The input image and the target image, which it should classify as real. The input image and the generated image (output of generator), which it should classify as fake. The discriminator loss function takes 2 inputs; real images, generated images real_loss is a sigmoid cross-entropy loss of the real images and generated_loss is a sigmoid cross-entropy loss of the generated images and Then the total_loss is the sum of real_loss and the generated_loss. The figure depicts the training procedure of the discriminator network. 


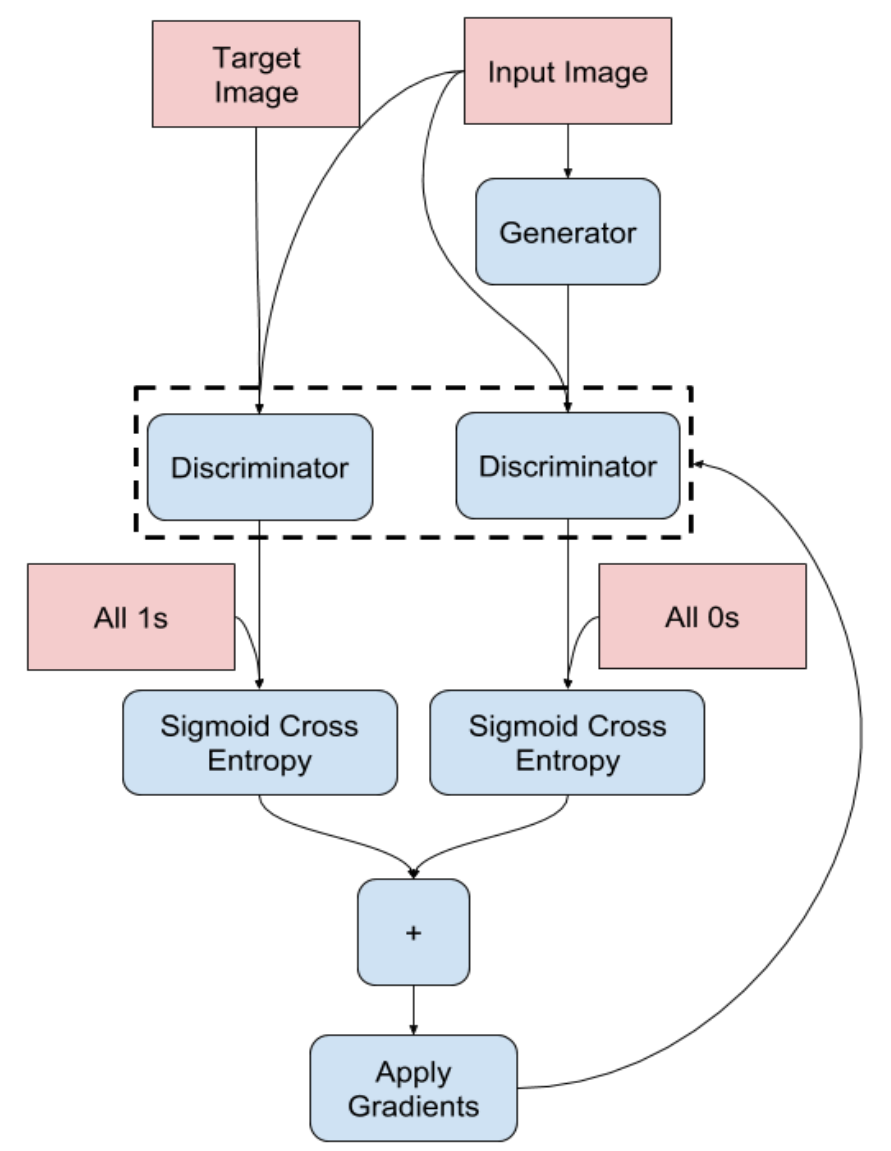

Figure. 11. The Training Procedure of Discriminator Network

\subsection{Loss Functions}

The generator network generates the new images using the random noise vector, to analyse the generated image quality. We measure the loss in generated results using Mean Squared Error (MSE) Metric is defined in Equation (5).

$$
\mathcal{L}_{M S E}(g t, g e n)=\ell_{M S E}(G(X g t), g e n)=\|G(g t)-g e n\|^{2}
$$

The Sigmoid Cross-Entropy loss comprises Sigmoid activation plus a Cross-Entropy loss. This loss functions are independent for each vector component (class), meaning that the loss computed for every CNN output vector component is not affected by other component values. That's why it is used for multi-label classification, were the insight of an element belonging to a certain class should not influence the decision for another class. It is called Binary Cross-Entropy Loss because it sets up a binary classification problems.

$$
\begin{gathered}
C E=-t_{1} \log \left(f\left(s_{1}\right)\right)-\left(1-t_{1}\right) \log \left(1-f\left(s_{1}\right)\right) \\
f\left(s_{i}\right)=\frac{1}{1+e^{-s_{i}}}
\end{gathered}
$$

\section{Experimental Results and discussion}

\subsection{The RWTH-PHOENIX-Weather 2014T dataset}

The RWTH-PHOENIX-Weather 2014T dataset [68] was collected from the phoenix Television channel for the years 2009 to 2011. The 386 editions based on weather forecast information have been prepared as a dataset. The dataset contains video clips, frames, and 
annotation details clearly in the corpus repository and available for free access. This dataset highly supports the development of a German Sign Language-based assistive system for speech loss and hearing loss people. It is a first created corpus for handling continuous sign language process at the sentence level. The videos are available with 210 X 260-pixel resolution and 25 frame rates per second. This dataset has been developed using 9 different signers and it consists of $7 \mathrm{k}$ sentences.

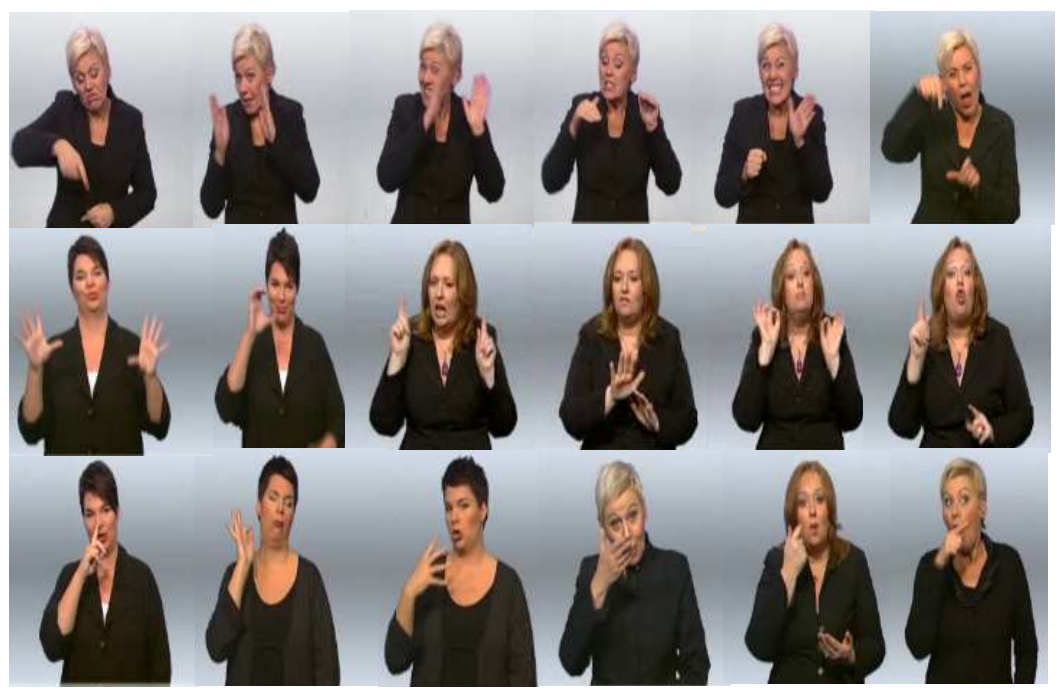

Figure. 12 The generated images of the RWTH-PHOENIX-Weather 2014T dataset using the proposed dynamicGAN model

\subsection{ISL-CSLTR: Indian Sign Language Dataset for Continuous Sign Language Translation and Recognition}

We created the ISL-CSLTR dataset [69] for Indian sign language. This corpus has been created to support the deaf-mute community. This dataset is freely accessible and research works on sign languages can utilize it. This novel corpus consists of 700 videos collected from 7 different signers with different background environments and luminance conditions. This corpus was primarily developed for handling hundred English sentences that are used frequently in daily life.

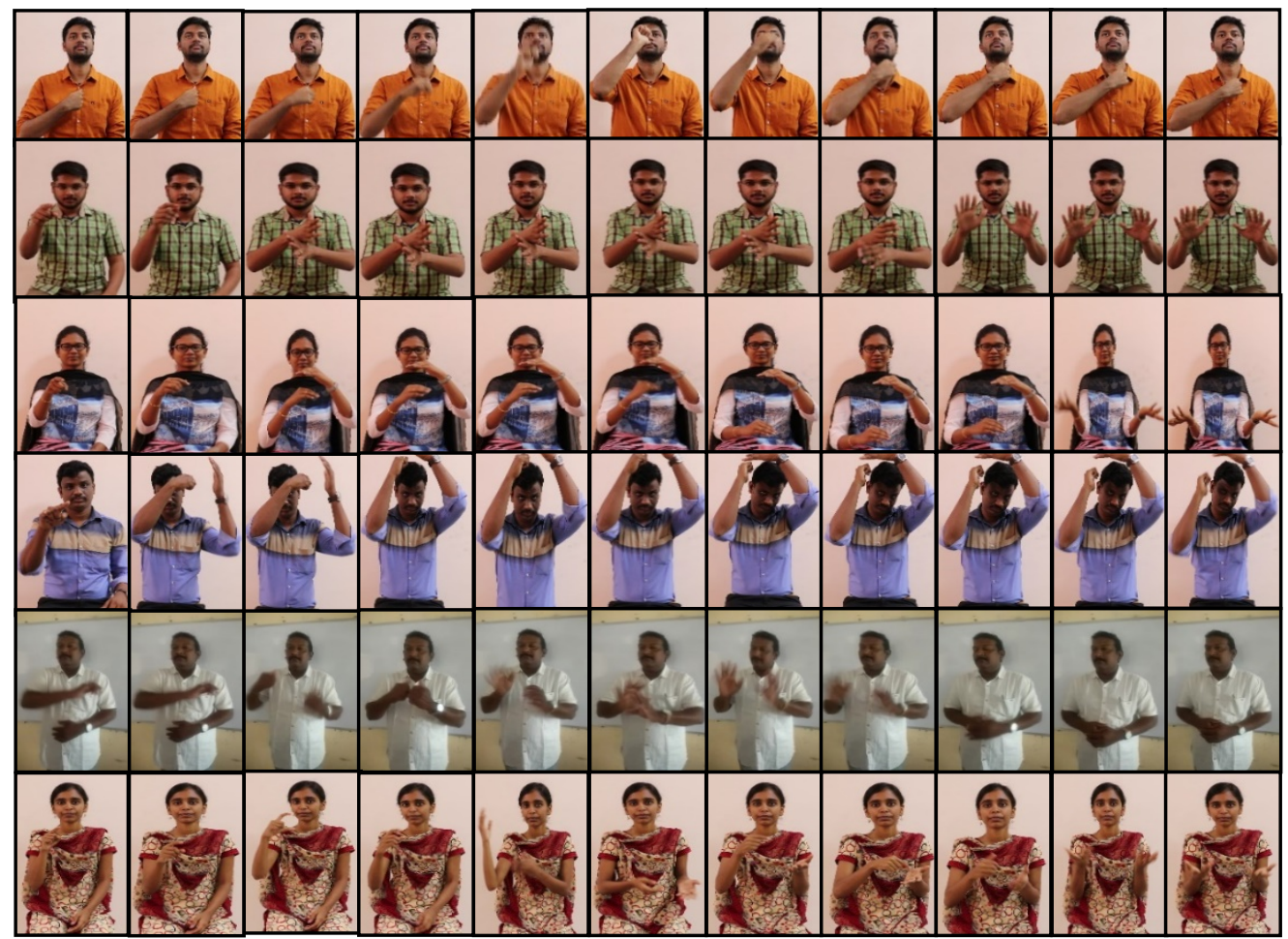


Figure. 13 The generated images of the ISL-CSLTR dataset using the proposed dynamicGAN model

\subsection{UCF101 - Action Recognition Data Set}

The UCF101 - Action Recognition Data Set [70] was collected from YouTube based on activities. This dataset has 101 different activity-based videos that project human daily life activities like applying makeup, playing the game, swimming, brushing, vegetable cutting, and typing.

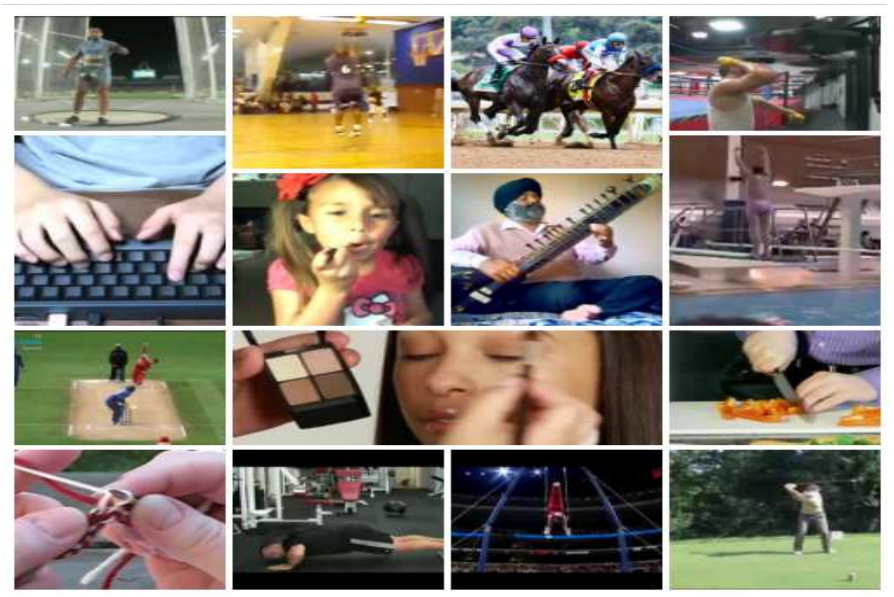

Figure. 14 The generated frames of the UCF101 - Action Recognition Data Set using the proposed dynamicGAN model

\subsection{Structural Similarity Index Measure (SSIM)}

The Structural Similarity Index Measure (SSIM) metric [56] used for assessing the image quality. We use the SSIM metric for comparing the model performance with existing approaches. This metric assesses the structural information degradation of generated video frames.

$$
l(x, y)=\frac{2 \mu_{x} \mu_{y}+C_{1}}{\mu_{x}^{2}+\mu_{y}^{2}+C_{1}}
$$

\begin{tabular}{|c|c|c|c|}
\hline \multirow{2}{*}{ Framework } & \multicolumn{3}{|c|}{ SSIM } \\
\cline { 2 - 4 } & $\begin{array}{c}\text { RWTH- } \\
\text { PHOENIX- } \\
\text { Weather 2014T } \\
\text { dataset }\end{array}$ & $\begin{array}{c}\text { ISL-CSLTR } \\
\text { dataset }\end{array}$ & $\begin{array}{c}\text { UCF101Action } \\
\text { Recognition } \\
\text { dataset }\end{array}$ \\
\hline MoCoGAN [91] & 0.702 & 0.802 & 0.856 \\
\hline Laplacian GAN [25] & 0.785 & 0.810 & 0.863 \\
\hline FutureGAN [92] & 0.852 & 0.826 & 0.796 \\
\hline VGAN [90] & 0.891 & 0.901 & 0.892 \\
\hline Deformable GAN [7] & 0.863 & 0.865 & 0.892 \\
\hline InfoGAN [3] & 0.836 & 0.796 & 0.783 \\
\hline 3CATN [18] & 0.785 & 0.693 & 0.782 \\
\hline Ours (DynamicGAN) & $\mathbf{0 . 9 0 1}$ & $\mathbf{0 . 9 3 7}$ & $\mathbf{0 . 9 2 5}$ \\
\hline
\end{tabular}

Table 2. The comparison of Structural Similarity Index Measure (SSIM) metric with proposed dynamic GAN model 


\subsection{Inception Score (IS)}

The proposed dynamicGAN model performance has experimented with inception score metrics. The high score denotes the model performance over multiple domains and the generation capability of the generator. The computation of IS is performed using the following equation.

$$
\operatorname{IS}(\mathcal{G})=\exp \left(\mathbb{E}_{x \sim p_{g}} \mathcal{D}_{K L}(p(y \mid x) \| p(y))\right)
$$

Let $\mathrm{x}$ denotes the generated images of the generator network $\mathrm{G}, p(y \mid x)$ denotes the class distribution of generated samples and the marginal probability function denoted as $p(y)$. The Inception score results are depicted in the table (2).

\begin{tabular}{|c|c|c|c|}
\hline \multirow{2}{*}{ Framework } & \multicolumn{3}{|c|}{ Inception Score } \\
\cline { 2 - 4 } & $\begin{array}{c}\text { RWTH-PHOENIX- } \\
\text { Weather 2014T } \\
\text { dataset }\end{array}$ & $\begin{array}{c}\text { ISL-CSLTR } \\
\text { dataset }\end{array}$ & $\begin{array}{c}\text { UCF101Action } \\
\text { Recognition dataset }\end{array}$ \\
\hline MoCoGAN [91] & 12.42 & 13.5 & 12.62 \\
\hline PG $^{2}$ GAN [6] & 14.23 & 14.3 & 13.23 \\
\hline FutureGAN [92] & 13.65 & 13.9 & 13.25 \\
\hline VGAN [90] & 13.2 & 13.1 & 13.0 \\
\hline Deformable GAN [7] & 12.68 & 12.36 & 13.6 \\
\hline InfoGAN [3] & 14.1 & 12.12 & 10.23 \\
\hline Pixel GAN [5] & 12.36 & 10.23 & 9.32 \\
\hline Ours (DynamicGAN) & $\mathbf{8 . 2}$ & $\mathbf{8 . 5}$ & $\mathbf{8 . 3}$ \\
\hline
\end{tabular}

Table 3. The comparison of Inception Score (IS) metric with proposed dynamic GAN model

\subsection{Peak Signal-to-Noise Ratio (PSNR)}

The generated video quality is evaluated using the PSNR quality metric. It compares the quality of generated results using ground truth images and provides the score. The higher PSNR value indicates improved quality in generated results. We compared our model performance with baseline models for the aforementioned three benchmark datasets and results are tabulated in table 5 where gt denotes the ground truth samples and gen denotes the generated results.

$$
\begin{aligned}
& \operatorname{PSNR}(\text { gt, gen })=10 \log _{10}\left(255^{2} / M S E(\text { gt, gen })\right) \\
& \operatorname{MSE}(\text { gt, gen })=\frac{1}{M N} \sum_{i=1}^{M} \sum_{j=1}^{N}\left(\mathrm{gt}_{\mathrm{ij}}-\mathrm{gen}_{\mathrm{ij}}\right)^{2}
\end{aligned}
$$

\begin{tabular}{|c|c|c|c|}
\hline \multirow{2}{*}{ Framework } & \multicolumn{3}{|c|}{ Inception Score } \\
\cline { 2 - 4 } & $\begin{array}{c}\text { RWTH-PHOENIX- } \\
\text { Weather 2014T } \\
\text { dataset }\end{array}$ & $\begin{array}{c}\text { ISL-CSLTR } \\
\text { dataset }\end{array}$ & $\begin{array}{c}\text { UCF101Action } \\
\text { Recognition dataset }\end{array}$ \\
\hline MoCoGAN [91] & 12.42 & 13.5 & 12.62 \\
\hline PG $^{2}$ GAN [6] & 14.23 & 14.3 & 13.23 \\
\hline FutureGAN [92] & 13.65 & 13.9 & 13.25 \\
\hline VGAN [90] & 13.2 & 13.1 & 13.0 \\
\hline Deformable GAN [7] & 12.68 & 12.36 & 13.6 \\
\hline InfoGAN [3] & 14.1 & 12.12 & 10.23 \\
\hline Pixel GAN [5] & 12.36 & 10.23 & $\mathbf{8 . 3 2}$ \\
\hline Ours (DynamicGAN) & $\mathbf{8 . 2}$ & $\mathbf{8 . 5}$ & \\
\hline
\end{tabular}

Table 4. The comparison of Inception Score (IS) metric with proposed dynamic GAN model 


\subsection{Fréchet Inception Distance (FID)}

The Fréchet Inception Distance (FID) metric [29] evaluates the generated video quality by considering the visual features and temporal details. Equation (6) is used for computing the FID of generated videos. D denotes the CNN model used to extract the features in the video. We use the VGG-19 model for feature extraction. $m_{r}, m_{f}$ denotes the mean values of features extracted from real and fake or generated samples, $\Sigma_{\mathrm{r}}, \Sigma_{\mathrm{f}}$ denotes covariance matrix of features from real and generated samples. The lowest score of FID is always better for video quality estimation.

$$
d^{2}\left(\left(m_{r}, \Sigma_{\mathrm{r}}\right),\left(m_{f}, \Sigma_{\mathrm{f}}\right)\right)=\|m r-m f\|_{2}^{2}+\operatorname{Tr}\left(\Sigma_{\mathrm{r}} \Sigma_{\mathrm{f}}-\left(2\left(\Sigma_{\mathrm{r}} \Sigma_{\mathrm{f}}\right)^{1 / 2}\right)\right)
$$

\begin{tabular}{|c|c|c|c|}
\hline \multirow{2}{*}{ Framework } & \multicolumn{3}{|c|}{ Fréchet Inception Distance (FID } \\
\cline { 2 - 4 } & $\begin{array}{c}\text { RWTH-PHOENIX- } \\
\text { Weather 2014T } \\
\text { dataset }\end{array}$ & $\begin{array}{c}\text { ISL-CSLTR } \\
\text { dataset }\end{array}$ & $\begin{array}{c}\text { UCF101Action } \\
\text { Recognition dataset }\end{array}$ \\
\hline MoCoGAN [91] & 36.42 & 13.5 & 12.62 \\
\hline PG'GAN [6] $^{2}$ G] & 33.23 & 14.3 & 13.23 \\
\hline FutureGAN [92] & 36.65 & 35.9 & 39.25 \\
\hline VGAN [90] & 36.2 & 34.1 & 41.0 \\
\hline Deformable GAN [7] & 28.68 & 37.36 & 39.6 \\
\hline InfoGAN [3] & 23.12 & 25.12 & 21.23 \\
\hline Pixel GAN [5] & 17.36 & 19.23 & 13.32 \\
\hline Ours (DynamicGAN) & $\mathbf{1 4 . 2}$ & $\mathbf{1 5 . 5}$ & $\mathbf{1 2 . 3}$ \\
\hline
\end{tabular}

Table 5. The comparison of the Fréchet Inception Distance (FID) metric with the proposed dynamic GAN model, shows the generated video quality of our model compared with baseline models.

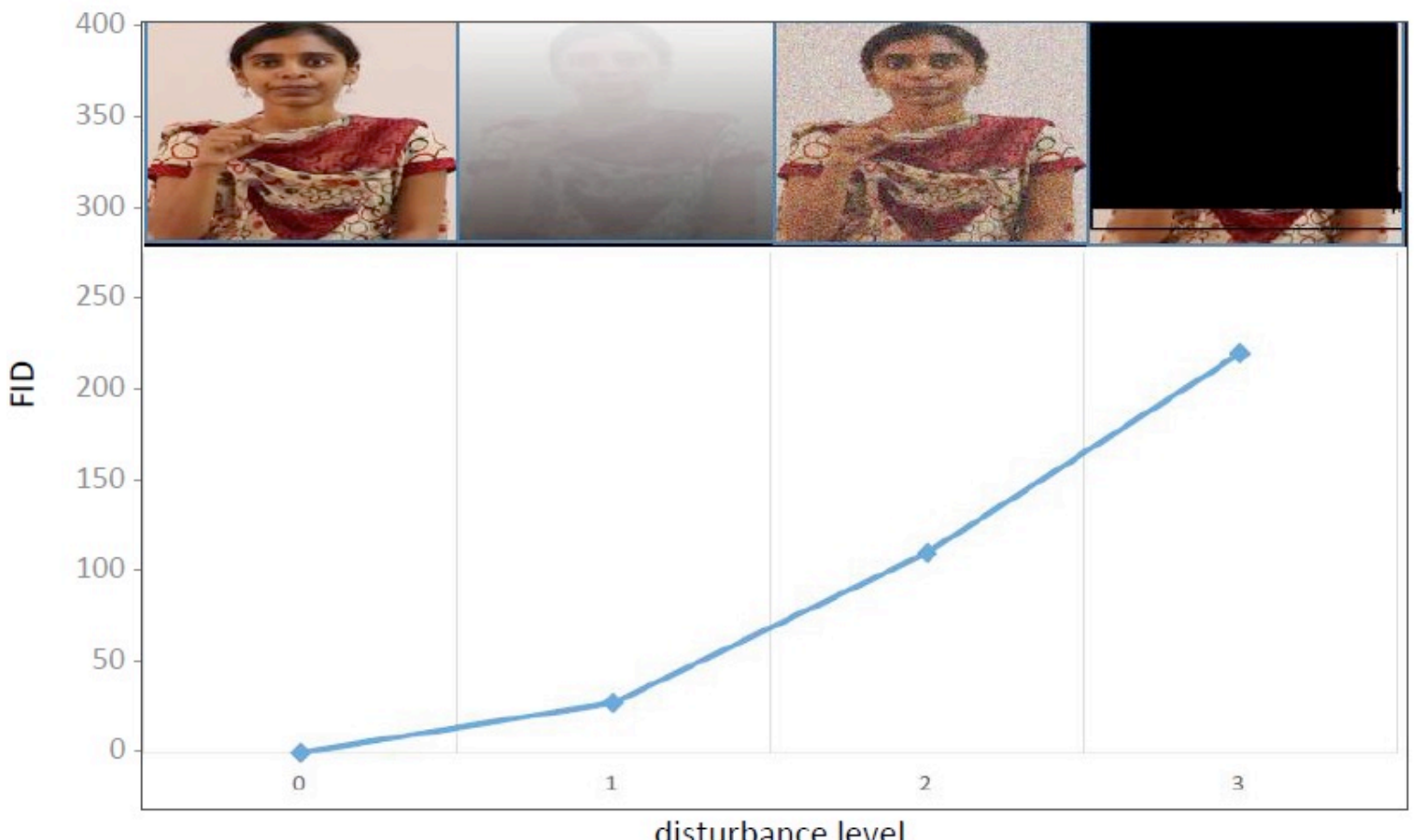

Figure 15: The Fréchet Inception Distance (FID) metric evaluates the generated samples at different noise and blurred levels. The monotonic increase of this evaluation captures the different disturbance levels. 


\section{Conclusion and Future work}

In this paper, the proposed dynamic GAN network introduces a novel method for unsupervised learning-based sign video generation from skeletal poses. The proposed work extends the basic GAN framework [1] for generating photo-realistic high-quality video generation. The model has experimented with benchmark datasets (i) RWTH-PHOENIXWeather 2014T dataset (ii) ISL-CSLTR and (iii) UCF101Action Recognition dataset. In our work, we have extended basic GAN architecture by applying different methods to address the challenges present in photo-realistic high-quality sign language video generation. We also evaluated the model performance with various metrics shows the improved performance over multiple domain-based datasets. These techniques can be effectively used in automating the spoken text to sign gesture translation task using mobile applications to serve the deaf-mute society.

\section{Compliance with Ethical Standards}

\section{Acknowledgments:}

We thank SASTRA Deemed University for providing infrastructural support to conduct the research. And also, we thank all the students for their contribution in collecting the sign videos and the successful completion of the ISL-CSLTR corpus. We would like to thank Navajeevan, Residential School for the Deaf, College of Spl. D.Ed \& B.Ed, Vocational Centre, and Child Care \& Learning Centre, Ayyalurimetta, Nandyal, Andhra Pradesh, India for their support and contribution in data acquisition.

\section{Funding Details}

The research was funded by the Science and Engineering Research Board (SERB), India under Start-up Research Grant (SRG)/2019-2021 (Grant no. SRG/2019/001338).

\section{Conflicts of interest}

The authors declare that there is no conflict of interest regarding the publication of this paper.

4. Informed Consent

No biological data or patients involved in this study.

\section{Contributions:}

Elakkiya $\mathrm{R}$ involved in problem formulation, data acquisition and network architecture design. Natarajan B involved in data analysis, data interpretation and model implementation. Both the authors contributed equally in drafting the manuscript.

\section{References}

[1] Goodfellow, Ian J., Jean Pouget-Abadie, Mehdi Mirza, Bing Xu, David Warde-Farley, Sherjil Ozair, Aaron Courville, and Yoshua Bengio. "Generative adversarial networks." arXiv preprint arXiv:1406.2661 (2014).

[2] Mirza, Mehdi, and Simon Osindero. "Conditional generative adversarial nets." arXiv preprint arXiv:1411.1784 (2014).

[3] Salimans, Tim, Ian Goodfellow, Wojciech Zaremba, Vicki Cheung, Alec Radford, and Xi Chen. "Improved techniques for training gans." arXiv preprint arXiv:1606.03498 (2016).

[4] Liu, Ming-Yu, and Oncel Tuzel. "Coupled generative adversarial networks." arXiv preprint arXiv:1606.07536 (2016).

[5] Isola, Phillip, Jun-Yan Zhu, Tinghui Zhou, and Alexei A. Efros. "Image-to-image translation with conditional adversarial networks." In Proceedings of the IEEE conference on computer vision and pattern recognition, pp. 1125-1134. 2017.

[6] Ma, Liqian, Xu Jia, Qianru Sun, Bernt Schiele, Tinne Tuytelaars, and Luc Van Gool. "Pose guided person image generation." arXiv preprint arXiv:1705.09368 (2017).

[7] Siarohin, Aliaksandr, Enver Sangineto, Stéphane Lathuiliere, and Nicu Sebe. "Deformable gans for pose-based human image generation." In Proceedings of the IEEE Conference on Computer Vision and Pattern Recognition, pp. 3408-3416. 2018.

[8] Elakkiya, R. "Machine learning based sign language recognition: a review and its research frontier." Journal of Ambient Intelligence and Humanized Computing (2020): 1-20.

[9] Saito, Yuki, Shinnosuke Takamichi, and Hiroshi Saruwatari. "Statistical parametric speech synthesis incorporating generative adversarial networks." IEEE/ACM Transactions on Audio, Speech, and Language Processing 26, no. 1 (2017): 84-96.

[10] Efros, Alexei A., and Thomas K. Leung. "Texture synthesis by non-parametric sampling." In Proceedings of the seventh IEEE international conference on computer vision, vol. 2, pp. 1033-1038. IEEE, 1999. 
[11] Shishir, Fairuz Shadmani, Tonmoy Hossain, and Faisal Muhammad Shah. "EsharaGAN: An Approach to Generate Disentangle Representation of Sign Language using InfoGAN." In 2020 IEEE Region 10 Symposium (TENSYMP), pp. 1383-1386. IEEE, 2020.

[12] Beschizza, Rob (2019-02-15). "This Person Does Not Exist". Boing-Boing. Retrieved 2019-02-16.

[13] Yang, Zhen, Wei Chen, Feng Wang, and Bo Xu. "Improving neural machine translation with conditional sequence generative adversarial nets." arXiv preprint arXiv:1703.04887 (2017).

[14] Li, Jingjing, Erpeng Chen, Zhengming Ding, Lei Zhu, Ke Lu, and Zi Huang. "Cycle-consistent conditional adversarial transfer networks." In Proceedings of the 27th ACM International Conference on Multimedia, pp. 747-755. 2019

[15] Mao, Xudong, Qing Li, Haoran Xie, Raymond YK Lau, Zhen Wang, and Stephen Paul Smolley. "Least squares generative adversarial networks." In Proceedings of the IEEE international conference on computer vision, pp. 2794-2802. 2017.

[16] Elakkiya, R., and K. Selvamani. "Subunit sign modeling framework for continuous sign language recognition." Computers \& Electrical Engineering 74 (2019): 379-390.

[17] Reed, Scott, Zeynep Akata, Xinchen Yan, Lajanugen Logeswaran, Bernt Schiele, and Honglak Lee. "Generative adversarial text to image synthesis." In International Conference on Machine Learning, pp. 10601069. PMLR, 2016.

[18] Zhang, Han, Tao Xu, Hongsheng Li, Shaoting Zhang, Xiaogang Wang, Xiaolei Huang, and Dimitris N. Metaxas. "Stackgan: Text to photo-realistic image synthesis with stacked generative adversarial networks." In Proceedings of the IEEE international conference on computer vision, pp. 5907-5915. 2017.

[19] Denton, Emily, Soumith Chintala, Arthur Szlam, and Rob Fergus. "Deep generative image models using a laplacian pyramid of adversarial networks." arXiv preprint arXiv:1506.05751 (2015).

[20] Choi, Yunjey, Minje Choi, Munyoung Kim, Jung-Woo Ha, Sunghun Kim, and Jaegul Choo. "Stargan: Unified generative adversarial networks for multi-domain image-to-image translation." In Proceedings of the IEEE conference on computer vision and pattern recognition, pp. 8789-8797. 2018.

[21] Choi, Yunjey, Youngjung Uh, Jaejun Yoo, and Jung-Woo Ha. "Stargan v2: Diverse image synthesis for multiple domains." In Proceedings of the IEEE/CVF Conference on Computer Vision and Pattern Recognition, pp. 8188-8197. 2020.

[22] Zhu, Jun-Yan, Taesung Park, Phillip Isola, and Alexei A. Efros. "Unpaired image-to-image translation using cycle-consistent adversarial networks." In Proceedings of the IEEE international conference on computer vision, pp. 2223-2232. 2017.

[23] Xu, Wenju, Shawn Keshmiri, and Guanghui Wang. "Adversarially approximated autoencoder for image generation and manipulation." IEEE Transactions on Multimedia 21, no. 9 (2019): 2387-2396.

[24] $\mathrm{Pu}$, Yunchen, Zhe Gan, Ricardo Henao, Xin Yuan, Chunyuan Li, Andrew Stevens, and Lawrence Carin. "Variational autoencoder for deep learning of images, labels and captions." arXiv preprint arXiv:1609.08976 (2016)

[25] Nie, Dong, Roger Trullo, Jun Lian, Caroline Petitjean, Su Ruan, Qian Wang, and Dinggang Shen. "Medical image synthesis with context-aware generative adversarial networks." In International conference on medical image computing and computer-assisted intervention, pp. 417-425. Springer, Cham, 2017.

[26] Wu, Hao, Jinghao Feng, Xuejin Tian, Fengyuan Xu, Yunxin Liu, XiaoFeng Wang, and Sheng Zhong. "secgan: A cycle-consistent gan for securely-recoverable video transformation." In Proceedings of the 2019 Workshop on Hot Topics in Video Analytics and Intelligent Edges, pp. 33-38. 2019.

[27] Karras, Tero, Samuli Laine, and Timo Aila. "A style-based generator architecture for generative adversarial networks." In Proceedings of the IEEE/CVF Conference on Computer Vision and Pattern Recognition, pp. 4401-4410. 2019.

[28] Xie, Zhaoheng, Reheman Baikejiang, Tiantian Li, Xuezhu Zhang, Kuang Gong, Mengxi Zhang, Wenyuan Qi, Evren Asma, and Jinyi Qi. "Generative adversarial network based regularized image reconstruction for PET." Physics in Medicine \& Biology 65, no. 12 (2020): 125016.

[29] Heusel, Martin, Hubert Ramsauer, Thomas Unterthiner, Bernhard Nessler, and Sepp Hochreiter. "Gans trained by a two time-scale update rule converge to a local nash equilibrium." arXiv preprint arXiv:1706.08500 (2017).

[30] Cui, Runpeng, Zhong Cao, Weishen Pan, Changshui Zhang, and Jianqiang Wang. "Deep Gesture Video Generation With Learning on Regions of Interest." IEEE Transactions on Multimedia 22, no. 10 (2019): 25512563.

[31] Stoll, Stephanie, Simon Hadfield, and Richard Bowden. "SignSynth: Data-Driven Sign Language Video Generation." In European Conference on Computer Vision, pp. 353-370. Springer, Cham, 2020.

[32] He, Jiawei, Andreas Lehrmann, Joseph Marino, Greg Mori, and Leonid Sigal. "Probabilistic video generation using holistic attribute control." In Proceedings of the European Conference on Computer Vision (ECCV), pp. 452-467. 2018

[33] Cai, Haoye, Chunyan Bai, Yu-Wing Tai, and Chi-Keung Tang. "Deep video generation, prediction and completion of human action sequences." In Proceedings of the European Conference on Computer Vision (ECCV), pp. 366-382. 2018.

[34] Clark, Aidan, Jeff Donahue, and Karen Simonyan. "Adversarial video generation on complex datasets." arXiv preprint arXiv:1907.06571 (2019).

[35] Wang, Tsun-Hsuan, Yen-Chi Cheng, Chieh Hubert Lin, Hwann-Tzong Chen, and Min Sun. "Point-to-point video generation." In Proceedings of the IEEE/CVF International Conference on Computer Vision, pp. 1049110500. 2019.

[36] Gao, Hang, Huazhe Xu, Qi-Zhi Cai, Ruth Wang, Fisher Yu, and Trevor Darrell. "Disentangling propagation and generation for video prediction." In Proceedings of the IEEE/CVF International Conference on Computer Vision, pp. 9006-9015. 2019. 
[37] Yan, Yichao, Jingwei Xu, Bingbing Ni, Wendong Zhang, and Xiaokang Yang. "Skeleton-aided articulated motion generation." In Proceedings of the 25th ACM international conference on Multimedia, pp. 199-207. 2017.

[38] Shan, Qi, Jiaya Jia, and Aseem Agarwala. "High-quality motion deblurring from a single image." Acm transactions on graphics (tog) 27, no. 3 (2008): 1-10.

[39] Gulrajani, Ishaan, Kundan Kumar, Faruk Ahmed, Adrien Ali Taiga, Francesco Visin, David Vazquez, and Aaron Courville. "Pixelvae: A latent variable model for natural images." arXiv preprint arXiv:1611.05013 (2016).

[40] Pathak, Deepak, Philipp Krahenbuhl, Jeff Donahue, Trevor Darrell, and Alexei A. Efros. "Context encoders: Feature learning by inpainting." In Proceedings of the IEEE conference on computer vision and pattern recognition, pp. 2536-2544. 2016.

[41] Wang, Chaoyue, Chang $\mathrm{Xu}$, Xin Yao, and Dacheng Tao. "Evolutionary generative adversarial networks." IEEE Transactions on Evolutionary Computation 23, no. 6 (2019): 921-934.

[42] Elakkiya, R., Kuppa Sai Sri Teja, L. Jegatha Deborah, Carmen Bisogni, and Carlo Medaglia. "Imaging based cervical cancer diagnostics using small object detection-generative adversarial networks." Multimedia Tools and Applications (2021): 1-17.

[43] Elakkiya, R., and K. Selvamani. "Enhanced dynamic programming approach for subunit modelling to handle segmentation and recognition ambiguities in sign language." Journal of Parallel and Distributed Computing 117 (2018): 246-255.

[44] Elakkiya, R., and K. Selvamani. "Extricating manual and non-manual features for subunit level medical sign modelling in automatic sign language classification and recognition." Journal of Medical Systems 41, no. 11 (2017): 1-13.

[45] Odena, Augustus, Christopher Olah, and Jonathon Shlens. "Conditional image synthesis with auxiliary classifier gans." In International conference on machine learning, pp. 2642-2651. PMLR, 2017.

[46] Arjovsky, Martin, Soumith Chintala, and Léon Bottou. "Wasserstein generative adversarial networks." In International conference on machine learning, pp. 214-223. PMLR, 2017.

[47] Karras, Tero, Timo Aila, Samuli Laine, and Jaakko Lehtinen. "Progressive growing of gans for improved quality, stability, and variation." arXiv preprint arXiv:1710.10196 (2017).

[48] Brock, Andrew, Jeff Donahue, and Karen Simonyan. "Large scale GAN training for high fidelity natural image synthesis." arXiv preprint arXiv:1809.11096 (2018).

[49] Mathieu, Michael, Camille Couprie, and Yann LeCun. "Deep multi-scale video prediction beyond mean square error." arXiv preprint arXiv:1511.05440 (2015).

[50] Dosovitskiy, Alexey, Jost Tobias Springenberg, and Thomas Brox. "Learning to generate chairs with convolutional neural networks." In Proceedings of the IEEE Conference on Computer Vision and Pattern Recognition, pp. 1538-1546. 2015.

[51] Radford, Alec, Luke Metz, and Soumith Chintala. "Unsupervised representation learning with deep convolutional generative adversarial networks." arXiv preprint arXiv:1511.06434 (2015).

[52] Cao, Zhe, Gines Hidalgo, Tomas Simon, Shih-En Wei, and Yaser Sheikh. "OpenPose: realtime multi-person 2D pose estimation using Part Affinity Fields." IEEE transactions on pattern analysis and machine intelligence 43, no. 1 (2019): 172-186.

[53] Pishchulin, Leonid, Mykhaylo Andriluka, Peter Gehler, and Bernt Schiele. "Poselet conditioned pictorial structures." In Proceedings of the IEEE Conference on Computer Vision and Pattern Recognition, pp. 588595. 2013.

[54] Dantone, Matthias, Juergen Gall, Christian Leistner, and Luc Van Gool. "Human pose estimation using body parts dependent joint regressors." In Proceedings of the IEEE Conference on Computer Vision and Pattern Recognition, pp. 3041-3048. 2013.

[55] Pfister, Tomas, James Charles, and Andrew Zisserman. "Flowing convnets for human pose estimation in videos." In Proceedings of the IEEE International Conference on Computer Vision, pp. 1913-1921. 2015.

[56] Wang, Zhou, Alan C. Bovik, Hamid R. Sheikh, and Eero P. Simoncelli. "Image quality assessment: from error visibility to structural similarity." IEEE transactions on image processing 13, no. 4 (2004): 600-612.

[57] Vondrick, Carl, Hamed Pirsiavash, and Antonio Torralba. "Generating videos with scene dynamics." arXiv preprint arXiv:1609.02612 (2016).

[58] Tulyakov, Sergey, Ming-Yu Liu, Xiaodong Yang, and Jan Kautz. "Mocogan: Decomposing motion and content for video generation." In Proceedings of the IEEE conference on computer vision and pattern recognition, pp. 1526-1535. 2018.

[59] Aigner, Sandra, and Marco Körner. "Futuregan: Anticipating the future frames of video sequences using spatio-temporal 3d convolutions in progressively growing gans." arXiv preprint arXiv:1810.01325 (2018).

[60] Chen, Xi, Yan Duan, Rein Houthooft, John Schulman, Ilya Sutskever, and Pieter Abbeel. "Infogan: Interpretable representation learning by information maximizing generative adversarial nets." arXiv preprint arXiv:1606.03657 (2016).

[61] Zhao, Bo, Xiao Wu, Zhi-Qi Cheng, Hao Liu, Zequn Jie, and Jiashi Feng. "Multi-view image generation from a single-view." In Proceedings of the 26th ACM international conference on Multimedia, pp. 383-391. 2018.

[62] Wang, Xiaolong, and Abhinav Gupta. "Generative image modeling using style and structure adversarial networks." In European conference on computer vision, pp. 318-335. Springer, Cham, 2016.

[63] Huang, Xun, Ming-Yu Liu, Serge Belongie, and Jan Kautz. "Multimodal unsupervised image-to-image translation." In Proceedings of the European conference on computer vision (ECCV), pp. 172-189. 2018.

[64] Zhang, Han, Ian Goodfellow, Dimitris Metaxas, and Augustus Odena. "Self-attention generative adversarial networks." In International conference on machine learning, pp. 7354-7363. PMLR, 2019.CVA 
[65] Larsen, Anders Boesen Lindbo, Søren Kaae Sønderby, Hugo Larochelle, and Ole Winther. "Autoencoding beyond pixels using a learned similarity metric." In International conference on machine learning, pp. 15581566. PMLR, 2016.

[66] Zhu, Jun-Yan, Richard Zhang, Deepak Pathak, Trevor Darrell, Alexei A. Efros, Oliver Wang, and Eli Shechtman. "Toward multimodal image-to-image translation." arXiv preprint arXiv:1711.11586 (2017).

[67] Kim, Taeksoo, Moonsu Cha, Hyunsoo Kim, Jung Kwon Lee, and Jiwon Kim. "Learning to discover crossdomain relations with generative adversarial networks." In International Conference on Machine Learning, pp. 1857-1865. PMLR, 2017.

[68] Koller, Oscar, Jens Forster, and Hermann Ney. "Continuous sign language recognition: Towards large vocabulary statistical recognition systems handling multiple signers." Computer Vision and Image Understanding 141 (2015): 108-125.

[69] R, Elakkiya; B, NATARAJAN (2021), "ISL-CSLTR: Indian Sign Language Dataset for Continuous Sign Language Translation and Recognition”, Mendeley Data, V1, doi: 10.17632/kcmpdxky7p.1

[70] Soomro, Khurram, Amir Roshan Zamir, and Mubarak Shah. "UCF101: A dataset of 101 human actions classes from videos in the wild." arXiv preprint arXiv:1212.0402 (2012).

[71] Liu, Ming-Yu, Xun Huang, Arun Mallya, Tero Karras, Timo Aila, Jaakko Lehtinen, and Jan Kautz. "Few-shot unsupervised image-to-image translation." In Proceedings of the IEEE/CVF International Conference on Computer Vision, pp. 10551-10560. 2019. 